International Journal of Modern Physics A

(C) World Scientific Publishing Company

\title{
Texture Zeros for Dirac Neutrinos and Current Experimental Tests
}

\author{
Xue-wen Liu \\ Department of Modern Physics \\ University of Science and Technology of China \\ Hefei 230026, China \\ xuewliu@mail.ustc.edu.cn \\ Shun Zhou \\ Department of Theoretical Physics \\ KTH Royal Institute of Technology \\ 10691 Stockholm, Sweden \\ shunzhou@kth.se \\ Received Day Month Year \\ Revised Day Month Year
}

\begin{abstract}
The Daya Bay and RENO reactor neutrino experiments have revealed that the smallest neutrino mixing angle is in fact relatively large, i.e., $\theta_{13} \approx 9^{\circ}$. Motivated by this exciting progress, we perform a systematic study of the neutrino mass matrix $M_{\nu}$ with one or two texture zeros, in the assumption that neutrinos are Dirac particles. Among fifteen possible patterns with two texture zeros, only three turn out to be favored by current neutrino oscillation data at the $3 \sigma$ level. Although all the six patterns with one texture zero are compatible with the experimental data at the $3 \sigma$ level, the parameter space of each pattern is strictly constrained. Phenomenological implications of $M_{\nu}$ on the leptonic $\mathrm{CP}$ violation and neutrino mass spectrum are explored, and the stability of texture zeros against the radiative corrections is also discussed.
\end{abstract}

Keywords: Texture Zeros; Neutrino Masses and Flavor Mixing; CP Violation.

PACS numbers: 14.60.Lm, 14.60.Pq

\section{Introduction}

The solar, atmospheric, accelerator and reactor neutrino experiments have provided us with compelling evidence that neutrinos are massive particles and they can transform from one flavor to another ${ }^{1}$ The lepton flavor mixing can be described by a $3 \times 3$ unitary matrix $U$, which is usually parameterized through three flavor mixing angles $\left(\theta_{12}, \theta_{23}, \theta_{13}\right)$ and one CP-violating phase $\delta$. To be explicit, we adopt the following parametrization

$$
U=\left(\begin{array}{ccc}
c_{12} c_{13} & s_{12} c_{13} & s_{13} \\
-c_{12} s_{23} s_{13}-s_{12} c_{23} e^{-i \delta} & -s_{12} s_{23} s_{13}+c_{12} c_{23} e^{-i \delta} & s_{23} c_{13} \\
-c_{12} c_{23} s_{13}+s_{12} s_{23} e^{-i \delta} & -s_{12} c_{23} s_{13}-c_{12} s_{23} e^{-i \delta} & c_{23} c_{13}
\end{array}\right),
$$


where $s_{i j} \equiv \sin \theta_{i j}$ and $c_{i j} \equiv \cos \theta_{i j}$ (for $i j=12,23,13$ ) have been defined. If neutrinos are Majorana particles, two additional $\mathrm{CP}$-violating phases $(\rho, \sigma)$ have to be introduced to fully describe the flavor mixing. Thanks to the elegant neutrino oscillation experiments, two neutrino mass-squared differences $\left(\delta m^{2}, \Delta m^{2}\right)$ and two flavor mixing angles $\left(\theta_{12}, \theta_{23}\right)$ have been measured with a reasonably good precision. More recently, the Daya Bay ${ }^{2}$ and $\mathrm{RENO}^{3}$ collaborations have clearly observed the disappearance of $\bar{\nu}_{e}$ from nuclear reactors, and revealed that the smallest mixing angle is relatively large, i.e., $\theta_{13} \approx 9^{\circ}$. This is really a great news to the longbaseline neutrino oscillation experiments, which aim to pin down the sign of $\Delta m^{2}$ and the magnitude of the $\mathrm{CP}$-violating phase $\delta$. In spite of the great progress made in neutrino physics, our understanding of neutrino properties is far from complete. For instance, the absolute scale of neutrino masses is not yet determined and whether neutrinos are Dirac or Majorana particles remains an open question.

Since a convincing flavor theory is lacking, the approach of texture zeros has been suggested to study the flavor problem for a long time. ${ }^{4}[8$ The texture zeros of a fermion mass matrix dynamically mean that the corresponding matrix elements are sufficiently suppressed in comparison with their neighboring counterparts, $\left.{ }^{9}\right]$ and they can help us to establish some simple and testable relations between flavor mixing angles and fermion mass ratios. $\frac{10] 12}{12}$ In fact, a great number of works have been devoted to confronting the zero textures of neutrino mass matrix with the neutrino oscillation data $\sqrt{13} \sqrt[52]{5}$ However, most of them have assumed neutrinos to be Majorana particles, because various seesaw mechanisms for neutrino mass generation lead to light Majorana neutrinos.

If neutrinos are Dirac particles, they can acquire masses exactly in the same way as quarks and charged leptons do in the standard model. In this scenario, it seems quite difficult to explain why the neutrino Yukawa couplings are twelve orders of magnitude smaller than the top-quark Yukawa coupling. Although this mass hierarchy problem has never been well understood even for charged fermions, it has been shown that the highly-suppressed Yukawa couplings for Dirac neutrinos can naturally be achieved in the models with extra spacial dimensions 5354 or through radiative mechanisms $[55$ Conservatively speaking, the most important motivation for considering Dirac neutrinos is the fact that no experiments have already excluded such a possibility. In the present work, we simply assume neutrinos to be Dirac particles and perform a systematic study of the neutrino mass matrix $M_{\nu}$ with one or two texture zeros.

Without loss of generality, we can take the mass matrix $M_{U}$ for Dirac neutrinos to be Hermitian by redefining the right-handed neutrino fields $\mathrm{a}$ As $M_{\nu}$ is Hermitian, three independent off-diagonal matrix elements are in general complex, while three independent diagonal ones are real. If $n$ of them are taken to be vanishing (i.e., $M_{\nu}$ 
has $n$ independent texture zeros), then we shall arrive at

$$
{ }^{6} \mathbf{C}_{n}=\frac{6 !}{n !(6-n) !}
$$

different textures. There are totally fifteen two-zero textures of $M_{\nu}$, which can be classified into six categories:

$$
\begin{aligned}
& \mathbf{A}_{1}:\left(\begin{array}{ccc}
0 & 0 & \triangle \\
0 & \times & \triangle \\
\triangle^{*} & \triangle^{*} & \times
\end{array}\right), \quad \mathbf{A}_{2}:\left(\begin{array}{ccc}
0 & \triangle & 0 \\
\triangle^{*} & \times & \triangle \\
0 & \triangle^{*} & \times
\end{array}\right) ; \\
& \mathbf{B}_{1}:\left(\begin{array}{ccc}
\times & \triangle & 0 \\
\triangle^{*} & 0 & \triangle \\
0 & \triangle^{*} & \times
\end{array}\right), \mathbf{B}_{2}:\left(\begin{array}{ccc}
\times & 0 & \triangle \\
0 & \times & \triangle \\
\triangle^{*} & \triangle^{*} & 0
\end{array}\right) \\
& \mathbf{B}_{3}:\left(\begin{array}{ccc}
\times & 0 & \triangle \\
0 & 0 & \triangle \\
\triangle^{*} & \triangle^{*} & \times
\end{array}\right), \mathbf{B}_{4}:\left(\begin{array}{ccc}
\times & \triangle & 0 \\
\triangle^{*} & \times & \triangle \\
0 & \triangle^{*} & 0
\end{array}\right) ; \\
& \mathbf{C}:\left(\begin{array}{ccc}
\times & \triangle & \triangle \\
\triangle^{*} & 0 & \triangle \\
\triangle^{*} & \triangle^{*} & 0
\end{array}\right) \\
& \mathbf{D}_{1}:\left(\begin{array}{ccc}
\times & \triangle & \triangle \\
\triangle^{*} & 0 & 0 \\
\triangle^{*} & 0 & \times
\end{array}\right), \quad \mathbf{D}_{2}:\left(\begin{array}{ccc}
\times & \triangle & \triangle \\
\triangle^{*} & \times & 0 \\
\triangle^{*} & 0 & 0
\end{array}\right) \\
& \mathbf{E}_{\mathbf{1}}:\left(\begin{array}{ccc}
0 & \triangle & \triangle \\
\triangle^{*} & 0 & \triangle \\
\triangle^{*} & \triangle^{*} & \times
\end{array}\right), \quad \mathbf{E}_{\mathbf{2}}:\left(\begin{array}{ccc}
0 & \triangle & \triangle \\
\triangle^{*} & \times & \triangle \\
\triangle^{*} & \triangle^{*} & 0
\end{array}\right), \quad \mathbf{E}_{\mathbf{3}}:\left(\begin{array}{ccc}
0 & \triangle & \triangle \\
\triangle^{*} & \times & 0 \\
\triangle^{*} & 0 & \times
\end{array}\right) ;
\end{aligned}
$$

and

$$
\mathbf{F}_{\mathbf{1}}:\left(\begin{array}{ccc}
\times & 0 & 0 \\
0 & \times & \triangle \\
0 & \triangle^{*} & \times
\end{array}\right), \quad \mathbf{F}_{\mathbf{2}}:\left(\begin{array}{ccc}
\times & 0 & \triangle \\
0 & \times & 0 \\
\triangle^{*} & 0 & \times
\end{array}\right), \quad \mathbf{F}_{\mathbf{3}}:\left(\begin{array}{ccc}
\times & \triangle & 0 \\
\triangle^{*} & \times & 0 \\
0 & 0 & \times
\end{array}\right),
$$

in which each " $\times$ " stands for a nonzero and real matrix element, while each " $\triangle$ " for a nonzero and complex one. Note that this classification is similar to that for the two-zero textures of Majorana neutrino mass matrix, $\frac{15}{18}$ which are symmetric and complex rather than Hermitian. Although the one-zero textures are in general less predictive than the two-zero ones, we shall consider them for completeness. Assuming one of six independent matrix elements to be zero, one can find out that there are six one-zero textures:

$$
\mathbf{P}_{1}:\left(\begin{array}{ccc}
0 & \triangle & \triangle \\
\triangle^{*} & \times & \triangle \\
\triangle^{*} & \triangle^{*} & \times
\end{array}\right), \quad \mathbf{P}_{2}:\left(\begin{array}{ccc}
\times & \triangle & \triangle \\
\triangle^{*} & 0 & \triangle \\
\triangle^{*} & \triangle^{*} & \times
\end{array}\right), \quad \mathbf{P}_{3}:\left(\begin{array}{ccc}
\times & \triangle & \triangle \\
\triangle^{*} & \times & \triangle \\
\triangle^{*} & \triangle^{*} & 0
\end{array}\right) ;
$$


and

$$
\mathbf{P}_{4}:\left(\begin{array}{ccc}
\times & 0 & \triangle \\
0 & \times & \triangle \\
\triangle^{*} & \triangle^{*} & \times
\end{array}\right), \quad \mathbf{P}_{5}:\left(\begin{array}{ccc}
\times & \triangle & 0 \\
\triangle^{*} & \times & \triangle \\
0 & \triangle^{*} & \times
\end{array}\right), \quad \mathbf{P}_{6}:\left(\begin{array}{ccc}
\times & \triangle & \triangle \\
\triangle^{*} & \times & 0 \\
\triangle^{*} & 0 & \times
\end{array}\right),
$$

where the notations are the same as those in Eqs. (3)-(8). It is straightforward to observe that three two-zero patterns $\mathbf{F}_{\mathbf{1 , 2}, \mathbf{3}}$ in Eq. (8) can be excluded, because they lead to just one nonzero flavor mixing angle.

We aim to confront the remaining twelve two-zero textures and six one-zero textures with the latest global-fit results of current neutrino oscillation data done by Fogli et al. in Ref. 63 b Only three two-zero textures, i.e., $\mathbf{A}_{\mathbf{1}}, \mathbf{A}_{2}$, and $\mathbf{C}$, are found to be compatible with current experimental data at the $3 \sigma$ level, so are all the six one-zero patterns, i.e., $\mathbf{P}_{i}$ (for $i=1,2, \cdots, 6$ ). In particular, most physical consequences of those viable patterns with one or two texture zeros have been explored in an analytical way, and the stability of texture zeros against radiative corrections is also discussed. We establish the relationship between the location of texture zeros and $\mathrm{CP}$ conservation, and demonstrate that the two-zero patterns $\mathbf{A}_{\mathbf{1 , 2}}$ and the one-zero patterns $\mathbf{P}_{\mathbf{4 , 5 , 6}}$ lead to CP conservation in the lepton sector.

The remaining parts of this paper are organized as follows. In section 2, we give some general remarks on the texture zeros for Dirac neutrinos. We show that it is possible to fully determine the neutrino mass spectrum and the CP-violating phase $\delta$ for all two-zero textures, as well as for the one-zero textures $\mathbf{P}_{\mathbf{4 , 5 , 6}}$. The relationship between the location of texture zeros and $\mathrm{CP}$ violation is pointed out. The stability of texture zeros for Dirac neutrino mass matrix against the renormalization group running is considered. Section 3 is devoted to the analytical and numerical analyses of the two-zero textures of $M_{\nu}$, while section 4 to the one-zero textures. Finally we summarize our conclusions in section 5 .

\section{General Remarks}

\subsection{Important relations}

In the flavor basis where the charged-lepton mass matrix $M_{l}$ is diagonal, the Dirac neutrino mass matrix $M_{\nu}$ can be reconstructed in terms of three neutrino masses $\left(m_{1}, m_{2}, m_{3}\right)$ and the flavor mixing matrix $U$. Namely,

$$
M_{\nu}=U\left(\begin{array}{ccc}
\lambda_{1} & 0 & 0 \\
0 & \lambda_{2} & 0 \\
0 & 0 & \lambda_{3}
\end{array}\right) U^{\dagger}
$$

where $\lambda_{1}=\eta \cdot m_{1}, \lambda_{2}=\chi \cdot m_{2}$ and $\lambda_{3}=m_{3}$ with $\eta, \chi= \pm 1$. Note that the three eigenvalues of a general $3 \times 3$ Hermitian matrix are real, but not necessarily positive,

\footnotetext{
${ }^{\mathrm{b}}$ The global-fit analysis of neutrino oscillation experiments has also been done in Refs. 64 and 65 however, all the results are completely consistent with each other at the $3 \sigma$ level. Therefore, our discussions will not be affected when the different global-fit results are used.
} 
so we have chosen the signs of the first two eigenvalues relative to the third one as $\eta$ and $\chi$. The parametrization of $U$ through three flavor mixing angles $\left(\theta_{12}, \theta_{23}, \theta_{13}\right)$ and one CP-violating phase $\delta$ has already been given in Eq. (1).

Now we explore the general consequences of the texture zeros of $M_{\nu}$. If one element of $M_{\nu}$ is vanishing, i.e., $\left(M_{\nu}\right)_{\alpha \beta}=0$, then we can obtain the corresponding constraint among the flavor mixing parameters

$$
\eta \cdot m_{1} U_{\alpha 1} U_{\beta 1}^{*}+\chi \cdot m_{2} U_{\alpha 2} U_{\beta 2}^{*}+m_{3} U_{\alpha 3} U_{\beta 3}^{*}=0 .
$$

Note that Eq. (12) implies one constraint condition in the case of $\alpha=\beta$ (e.g., the one-zero textures $\mathbf{P}_{\mathbf{1 , 2 , 3}}$ ), but two constraint conditions in the case of $\alpha \neq \beta$ (e.g., the one-zero textures $\left.\mathbf{P}_{\mathbf{4 , 5 , 6}}\right)$. In the former case, one can derive from Eq. (12) that either $\eta=\chi=-1$ or $\eta \cdot \chi=-1$ must hold and

$$
\left|U_{\alpha 2}\right|^{2}=\frac{1}{1-\chi \zeta}-\frac{1-\eta \xi}{1-\chi \zeta} \cdot\left|U_{\alpha 1}\right|^{2},
$$

where $\xi \equiv m_{1} / m_{3}$ and $\zeta \equiv m_{2} / m_{3}$ have been defined. In the latter case, we can get two constraint conditions by requiring both real and imaginary parts of the left-hand side of Eq. (12) to be zero. More explicitly, we have

$$
(\eta \xi-\chi \zeta) \cdot \operatorname{Im}\left[K_{23}^{\alpha \beta}\right]=0,
$$

and

$$
\operatorname{Re}\left[K_{23}^{\alpha \beta}\right]=-\frac{1-\eta \xi}{1-\chi \zeta} \cdot \operatorname{Re}\left[K_{13}^{\alpha \beta}\right],
$$

where $K_{i j}^{\alpha \beta} \equiv U_{\alpha i} U_{\alpha j}^{*} U_{\beta i}^{*} U_{\beta j}$. As will be shown later, the relations in Eqs. (13), (14) and (15) are very useful in the determination of flavor mixing parameters when we discuss the one-zero textures in section 4.

If two independent elements of $M_{\nu}$ are vanishing, i.e., $\left(M_{\nu}\right)_{a b}=\left(M_{\nu}\right)_{\alpha \beta}=0$ with $a b \neq \alpha \beta$ as shown in Eqs. (3)-(8), we can obtain

$$
\begin{aligned}
& \xi \equiv \frac{m_{1}}{m_{3}}=\eta \cdot \frac{U_{a 3} U_{b 3}^{*} U_{\alpha 2} U_{\beta 2}^{*}-U_{a 2} U_{b 2}^{*} U_{\alpha 3} U_{\beta 3}^{*}}{U_{a 2} U_{b 2}^{*} U_{\alpha 1} U_{\beta 1}^{*}-U_{a 1} U_{b 1}^{*} U_{\alpha 2} U_{\beta 2}^{*}}, \\
& \zeta \equiv \frac{m_{2}}{m_{3}}=\chi \cdot \frac{U_{a 1} U_{b 1}^{*} U_{\alpha 3} U_{\beta 3}^{*}-U_{a 3} U_{b 3}^{*} U_{\alpha 1} U_{\beta 1}^{*}}{U_{a 2} U_{b 2}^{*} U_{\alpha 1} U_{\beta 1}^{*}-U_{a 1} U_{b 1}^{*} U_{\alpha 2} U_{\beta 2}^{*}} .
\end{aligned}
$$

Since both $\xi$ and $\zeta$ are by definition real and non-negative, the imaginary parts of the quantities on the right-hand side of Eq. (16) have to disappear. This requirement may lead us to the determination of the CP-violating phase $\delta$, as we shall show below.

\subsection{Texture zeros and CP violation}

Now that $\theta_{13}$ has been measured to be relatively large, the CP-violating effects are promising to be discovered in the long-baseline neutrino oscillation experiments, if the CP-violating phase $\delta$ turns out to be not extremely small or very close to $\pi$. As 
is well known, the $\mathrm{CP}$ violation is characterized by the Jarlskog invariant $\mathcal{J}$, which is defined as 66 67

$$
\operatorname{Im}\left[K_{i j}^{\alpha \beta}\right] \equiv \mathcal{J} \cdot \sum_{\gamma} \epsilon_{\alpha \beta \gamma} \sum_{k} \epsilon_{i j k},
$$

where $\epsilon_{\alpha \beta \gamma}$ and $\epsilon_{i j k}$ denote the Levi-Civita symbol, and $K_{i j}^{\alpha \beta}$ has been defined below Eq. (15). It is now straightforward to discuss the $\mathrm{CP}$ violation for the zero textures. Some comments are in order:

- $C P$-violating one-zero textures - In the case of $\left(M_{\nu}\right)_{\alpha \beta}=0$ with $\alpha \neq \beta$, Eq. (14) implies either $\eta \xi=\chi \zeta$ or $\operatorname{Im}\left[K_{23}^{\alpha \beta}\right]= \pm \mathcal{J}=0$. Note that the solar neutrino experiments have established $m_{2}>m_{1}$, or equivalently $\zeta>\xi$, so we are left with $\mathcal{J}=0$. Therefore, $\mathrm{CP}$ violation is only possible for the patterns $\mathbf{P}_{\mathbf{1 , 2 , 3}}$, and we have $\delta=0$ or $\pi$ for the other one-zero patterns.

- $C P$-violating two-zero textures - Note that Eqs. (13), (14) and (15) apply as well to the two-zero textures. Hence $\mathrm{CP}$ violation is only possible for the textures with both $a=b$ and $\alpha=\beta$. In other words, the patterns $\mathbf{C}, \mathbf{E}_{\mathbf{1}}$, $\mathbf{E}_{2}$ can lead to leptonic CP violation, while $\delta=0$ or $\pi$ holds for all the other two-zero textures.

In order to demonstrate the above observation of CP-violating two-zero textures, we can directly calculate the imaginary part of the first identity in Eq. (16). It turns out that

$\operatorname{Re}\left[K_{32}^{a b}\right] \cdot \operatorname{Im}\left[K_{21}^{\alpha \beta}\right]+\operatorname{Im}\left[K_{32}^{a b}\right] \cdot \operatorname{Re}\left[K_{21}^{\alpha \beta}\right]-\left|U_{a 2}\right|^{2}\left|U_{b 2}\right|^{2} \cdot \operatorname{Im}\left[K_{31}^{\alpha \beta}\right]+(a b \leftrightarrow \alpha \beta)=0$,

where " $a b \leftrightarrow \alpha \beta$ " stands for the foregoing terms with the exchange of $a \leftrightarrow \alpha$ and $b \leftrightarrow \beta$ both in the superscripts and in the subscripts. Since the patterns $\mathbf{F}_{\mathbf{1 , 2 , \mathbf { 3 }}}$ have already been excluded, we need to consider only two different cases: (1) $a=b$ and $\alpha=\beta$; (2) $a=b$ and $\alpha \neq \beta$. In the first case, it is easy to see that the quantities on the right-hand side of Eq. (16) are automatically real, so there is no constraint on the CP-violating phase. In the second case, Eq. (18) reduces to $\operatorname{Im}\left[K_{31}^{\alpha \beta}\right]= \pm \mathcal{J}=0$, which is consistent with our previous observation. Note that the result for the second identity in Eq. (16) can be obtained by exchanging the subscripts "1" and "2" in Eq. (18), however, it doesn't give any new constraints. Hence we can conclude that only one off-diagonal texture zero in the Hermitian Dirac neutrino mass matrix is enough to ensure $\mathrm{CP}$ conservation in the lepton sector.

\subsection{Parameter counting}

Since we have assumed massive neutrinos to be Dirac particles, there are seven physical parameters: three neutrino masses $\left(m_{1}, m_{2}, m_{3}\right)$, three flavor mixing angles $\left(\theta_{12}, \theta_{23}, \theta_{13}\right)$, and one CP-violating phase $\delta$. The number of constraint relations caused by the texture zeros depends on the location of zeros, so we count the parameters for four distinct situations: 
(1) One off-diagonal zero - This category contains the patterns $\mathbf{P}_{\mathbf{4 , 5 , 6}}$. As shown in Eqs. (14) and (15), there are two independent constraint relations. Therefore, with the help of current experimental measurements of three flavor mixing angles $\left(\theta_{12}, \theta_{23}, \theta_{13}\right)$ and two neutrino mass-squared differences, defined as $s^{63}$

$$
\delta m^{2} \equiv m_{2}^{2}-m_{1}^{2}, \quad \Delta m^{2}=m_{3}^{2}-\frac{1}{2}\left(m_{1}^{2}+m_{2}^{2}\right),
$$

we can fully determine the neutrino mass spectrum and the CP-violating phase. As shown in section 2.2, these patterns predict CP conservation and thus $\delta=0$ or $\pi$. Using Eq. (15) and the following relation

$$
R_{\nu} \equiv \frac{\delta m^{2}}{\left|\Delta m^{2}\right|}=\frac{2\left(\zeta^{2}-\xi^{2}\right)}{\left|2-\left(\zeta^{2}+\xi^{2}\right)\right|}
$$

we can fix $\xi$ and $\zeta$. Thus three neutrino masses are given by

$$
m_{3}=\frac{\sqrt{\delta m^{2}}}{\sqrt{\zeta^{2}-\xi^{2}}}, \quad m_{2}=m_{3} \zeta, \quad m_{1}=m_{3} \xi .
$$

The detailed discussions of $\mathbf{P}_{\mathbf{4 , 5 , 6}}$ will be given in section 4 .

(2) One diagonal zero - This category contains the patterns $\mathbf{P}_{\mathbf{1 , 2 , 3}}$. The texture zero leads to one constraint condition in Eq. (13). Given the five experimental observables, one parameter is left free for these patterns. If the CP-violating phase is fixed, we can pin down the neutrino mass spectrum by using Eqs. (13) and (20). On the other hand, if the lightest neutrino mass is assumed, one can determine the CP-violating phase.

(3) One diagonal zero and one off-diagonal zero - This category contains the patterns $\mathbf{A}_{\mathbf{1}, \mathbf{2}}, \mathbf{B}_{\mathbf{1 , 2 , 3}, \mathbf{4}}, \mathbf{D}_{\mathbf{1 , 2}}$, and $\mathbf{E}_{\mathbf{3}}$. These two texture zeros impose three constraint conditions, so both three neutrino masses and the CP-violating phase can be calculated by using experimental observables. Since these patterns are $\mathrm{CP}$ conserving, we have $\delta=0$ or $\pi$. Therefore, $\xi$ and $\zeta$ can be calculated from Eq. (16), and the neutrino mass spectrum is then given by Eq. (21). Note that $\xi$ and $\zeta$ have to satisfy Eq. (20), so there will be one testable correlative relation among the flavor mixing angles and neutrino masses.

(4) Two diagonal zeros - These category contains the patterns $\mathbf{C}$ and $\mathbf{E}_{\mathbf{1 , 2}}$. The texture zeros induce two constraint relations, which together with five experimental observables leads to the full determination of neutrino mass spectrum and the CP-violating phase. This can be done as follows. First, note that $\xi$ and $\zeta$ are functions of the CP-violating phase $\delta$ as shown in Eq. (16). Then it is possible to determine or constrain $\delta$ from Eq. (20). Once $\delta$ is fixed, we can obtain $(\xi, \zeta)$ from Eq. (16), and thus neutrino masses from Eq. (21). All the two-zero textures will be discussed in great detail in section 3 .

Now we summarize the latest global-fit results of three flavor mixing angles $\left(\theta_{12}, \theta_{23}, \theta_{13}\right)$ and two neutrino mass-squared differences $\left(\delta m^{2}, \Delta m^{2}\right)$, which will be 
taken as experimental observables to determine the other flavor parameters. For the normal mass hierarchy with $\Delta m^{2}>0$, it has been found at the $3 \sigma$ level ${ }^{\sqrt{63}}$

$$
\begin{aligned}
& 0.259 \leq \sin ^{2} \theta_{12} \leq 0.359 \quad \text { or } \quad 30.6^{\circ} \leq \theta_{12} \leq 36.8^{\circ}, \\
& 0.331 \leq \sin ^{2} \theta_{23} \leq 0.637 \quad \text { or } \quad 35.1^{\circ} \leq \theta_{23} \leq 53.0^{\circ}, \\
& 0.017 \leq \sin ^{2} \theta_{13} \leq 0.031 \quad \text { or } \quad 7.5^{\circ} \leq \theta_{13} \leq 10.1^{\circ}
\end{aligned}
$$

and

$$
\begin{aligned}
& 6.99 \times 10^{-5} \mathrm{eV}^{2} \leq \delta m^{2} \leq 8.18 \times 10^{-5} \mathrm{eV}^{2}, \\
& 2.19 \times 10^{-3} \mathrm{eV}^{2} \leq+\Delta m^{2} \leq 2.62 \times 10^{-3} \mathrm{eV}^{2} .
\end{aligned}
$$

For the inverted mass hierarchy with $\Delta m^{2}<0$, the global analysis yields $s^{63}$

$$
\begin{aligned}
& 0.259 \leq \sin ^{2} \theta_{12} \leq 0.359 \quad \text { or } \quad 30.6^{\circ} \leq \theta_{12} \leq 36.8^{\circ}, \\
& 0.335 \leq \sin ^{2} \theta_{23} \leq 0.663 \quad \text { or } \quad 35.4^{\circ} \leq \theta_{23} \leq 54.5^{\circ}, \\
& 0.017 \leq \sin ^{2} \theta_{13} \leq 0.032 \quad \text { or } \quad 7.5^{\circ} \leq \theta_{13} \leq 10.3^{\circ} ;
\end{aligned}
$$

and

$$
\begin{aligned}
& 6.99 \times 10^{-5} \mathrm{eV}^{2} \leq \delta m^{2} \leq 8.18 \times 10^{-5} \mathrm{eV}^{2}, \\
& 2.17 \times 10^{-3} \mathrm{eV}^{2} \leq-\Delta m^{2} \leq 2.61 \times 10^{-3} \mathrm{eV}^{2},
\end{aligned}
$$

at the $3 \sigma$ level. The best-fit values of three mixing angles are $\theta_{12}=33.6^{\circ}, \theta_{23}=$ $38.4^{\circ}$, and $\theta_{13}=8.9^{\circ}$, while those of neutrino mass-squared differences are $\delta m^{2}=$ $7.54 \times 10^{-5} \mathrm{eV}^{2}$ and $\Delta m^{2}=2.43 \times 10^{-3} \mathrm{eV}^{2}$. It is interesting to note that the best-fit value of the CP-violating phase is $\delta \sim \pi$, which happens to be consistent with the prediction of the Dirac mass matrix with one off-diagonal texture zero. However, there is no constraint on $\delta$ at the $3 \sigma$ level.

\subsection{Stability of texture zeros}

The stability of texture zeros for Majorana neutrinos has already been discussed in the literature, ${ }^{44}\left[68 \mid 69\right.$ by using the renormalization-group equations (RGEs) ${ }^{70 \mid 71} \mathrm{It}$ has been demonstrated that the texture zeros of Majorana neutrino mass matrix are stable against the one-loop quantum corrections ${ }^{44}$ In this subsection, we shall examine whether the stability of texture zeros for Dirac neutrinos is maintained $\left[\frac{52}{5}\right.$

To accommodate Dirac neutrino masses, one can extend the standard model with three right-handed neutrino singlets, and simply require the lepton number conservation to forbid the Majorana neutrino mass term. At the one-loop level, the RGEs for Dirac neutrinos and charged leptons can be written as ${ }^{72}$

$$
\begin{aligned}
16 \pi^{2} \frac{\mathrm{d} Y_{\nu}}{\mathrm{d} t} & =\left[\alpha_{\nu}+C_{\nu}^{\nu}\left(Y_{\nu} Y_{\nu}^{\dagger}\right)+C_{\nu}^{l}\left(Y_{l} Y_{l}^{\dagger}\right)\right] Y_{\nu}, \\
16 \pi^{2} \frac{\mathrm{d} Y_{l}}{\mathrm{~d} t} & =\left[\alpha_{l}+C_{l}^{\nu}\left(Y_{\nu} Y_{\nu}^{\dagger}\right)+C_{l}^{l}\left(Y_{l} Y_{l}^{\dagger}\right)\right] Y_{l},
\end{aligned}
$$


where $t \equiv \ln \left(\mu / M_{Z}\right)$ with $\mu$ being an arbitrary renormalization scale, and $M_{Z}$ the $Z$-boson mass. Here $Y_{\nu, l}=\sqrt{2} M_{\nu, l} / v$ denotes respectively the neutrino and chargedlepton Yukawa coupling matrices, and $v \approx 246 \mathrm{GeV}$ is the vacuum expectation value of the Higgs field.

In the standard model, the relevant coefficients are $C_{\nu}^{\nu}=C_{l}^{l}=+3 / 2, C_{\nu}^{l}=C_{l}^{\nu}=$ $-3 / 2, \alpha_{\nu}=-9 g_{1}^{2} / 20-9 g_{2}^{2} / 4+T$ and $\alpha_{l}=-9 g_{1}^{2} / 4-9 g_{2}^{2} / 4+T$, where $g_{1}$ and $g_{2}$ are gauge couplings, and $T=\operatorname{Tr}\left[3\left(Y_{\mathrm{u}} Y_{\mathrm{u}}^{\dagger}\right)+3\left(Y_{\mathrm{d}} Y_{\mathrm{d}}^{\dagger}\right)+\left(Y_{\nu} Y_{\nu}^{\dagger}\right)+\left(Y_{l} Y_{l}^{\dagger}\right)\right]$ with $Y_{\mathrm{q}}$ (for $\mathrm{q}=\mathrm{u}, \mathrm{d}$ ) being the up-type and down-type quark Yukawa coupling matrices. The term $Y_{\nu} Y_{\nu}^{\dagger}$ in Eq. (26) can be safely neglected, because neutrino masses are much smaller than charged-lepton masses. In the flavor basis where the chargedlepton Yukawa matrix is diagonal $Y_{l}=\operatorname{Diag}\left\{y_{e}, y_{\mu}, y_{\tau}\right\}$, one can observe from the second identity in Eq. (26) that the charged-lepton Yukawa coupling matrix remains diagonal as the energy scale evolves. After solving the RGE for $Y_{\nu}$, one can find that the neutrino mass matrix at the weak scale $M_{Z}$ is related to that at a high-energy scale $\Lambda$ in the following way

$$
M_{\nu}\left(M_{Z}\right)=I_{0}\left(\begin{array}{ccc}
I_{e} & 0 & 0 \\
0 & I_{\mu} & 0 \\
0 & 0 & I_{\tau}
\end{array}\right) M_{\nu}(\Lambda)
$$

where the RGE evolution function $I_{0}$ represents the overall contribution from gauge and quark Yukawa couplings, while $I_{\alpha}$ (for $\alpha=e, \mu, \tau$ ) stand for the contributions from charged-lepton Yukawa couplings. Because of $m_{e} \ll m_{\mu} \ll m_{\tau}$, we have $I_{e}<I_{\mu}<I_{\tau}$ and they will modify the structure of $M_{\nu}$. In contrast, $I_{0} \neq 1$ just affects the absolute scale of neutrino masses. However, the texture zeros of $M_{\nu}$ are stable against the one-loop RGE corrections. Taking the pattern $\mathbf{A}_{\mathbf{1}}$ for example, we have

$$
M_{\nu}^{\mathbf{A}_{1}}(\Lambda)=\left(\begin{array}{ccc}
0 & 0 & a \\
0 & b & c \\
a^{*} & c^{*} & d
\end{array}\right)
$$

at $\Lambda$, and thus

$$
M_{\nu}^{\mathbf{A}_{1}}\left(M_{Z}\right)=I_{0}\left(\begin{array}{ccc}
0 & 0 & a I_{e} \\
0 & b I_{\mu} & c I_{\mu} \\
a^{*} I_{\tau} & c^{*} I_{\tau} & d I_{\tau}
\end{array}\right)
$$

at $M_{Z}$. Note that the neutrino matrix $M_{\nu}^{\mathbf{A}_{1}}\left(M_{Z}\right)$ is no longer exactly Hermitian. Nevertheless, it can be shown that $I_{\alpha} \approx 1$ (for $\alpha=e, \mu, \tau$ ) hold as an excellent approximation in the standard model. As a consequence, the previous important relations derived from texture zeros are formally valid both at $\Lambda$ and $M_{Z}$, at least in the lowest-order approximation. 


\section{Two-zero Textures}

\subsection{Analytical approximations}

First of all, we consider the two-zero textures and explore their phenomenological implications in an analytical way. It has been demonstrated that a permutation symmetry between the viable two-zero patterns $\mathbf{A}_{\mathbf{1}}$ and $\mathbf{A}_{\mathbf{2}}, \mathbf{B}_{\mathbf{1}}$ and $\mathbf{B}_{\mathbf{2}}$, or $\mathbf{B}_{\mathbf{3}}$ and $\mathbf{B}_{\mathbf{4}}$ for Majorana neutrinos. 44 The existence of such a symmetry originates from the fact that the location of texture zeros in each pair is related by an exchange between the last two rows and columns of $M_{\nu}$. For the same reason, it is only necessary to study the patterns $\mathbf{A}_{\mathbf{1}}, \mathbf{B}_{\mathbf{1}}, \mathbf{B}_{\mathbf{3}}, \mathbf{D}_{\mathbf{1}}, \mathbf{E}_{\mathbf{1}}$ and $\mathbf{E}_{\mathbf{3}}$ of Dirac neutrino mass matrix, and the results for the other patterns can be obtained with the replacements $\theta_{23} \rightarrow \pi / 2-\theta_{23}$ and $\delta \rightarrow \delta-\pi$. Now we discuss these patterns by making some reasonable approximations.

- Pattern $\mathbf{A}_{1}$ with $\left(M_{\nu}\right)_{e e}=\left(M_{\nu}\right)_{e \mu}=0$. As we have discussed, there is no CP violation and thus $\delta=0$ or $\pi$. In the case of $\delta=0$, we obtain from Eq. (16) that

$$
\begin{aligned}
& \xi=+\eta \cdot \frac{s_{13}}{c_{13}^{2}}\left(\frac{s_{12} s_{23}}{c_{12} c_{23}}-s_{13}\right), \\
& \zeta=-\chi \cdot \frac{s_{13}}{c_{13}^{2}}\left(\frac{c_{12} s_{23}}{s_{12} c_{23}}+s_{13}\right) .
\end{aligned}
$$

Obviously, $\eta=+1$ and $\chi=-1$ should be taken to ensure that $\xi$ and $\zeta$ are non-negative. Since $s_{13}^{2} \ll 1$ still holds, Eq. (30) approximates to

$$
\begin{aligned}
& \xi \approx \sin \theta_{13} \tan \theta_{23} \tan \theta_{12}, \\
& \zeta \approx \sin \theta_{13} \tan \theta_{23} \cot \theta_{12} .
\end{aligned}
$$

Given $0.59 \leq \tan \theta_{12} \leq 0.75,0.70 \leq \tan \theta_{23} \leq 1.3$, and $0.13 \leq \sin \theta_{13} \leq 0.18$ at the $3 \sigma$ level, Eq. (31) leads to $\xi<\zeta<1$. Hence only the normal neutrino mass hierarchy is allowed, i.e., $\Delta m^{2}>0$. Taking the best-fit values of three neutrino mixing angles (i.e., $\theta_{12}=33.6^{\circ}, \theta_{23}=38.4^{\circ}$, and $\theta_{13}=8.9^{\circ}$ ) and those of two neutrino mass-squared differences (i.e., $\delta m^{2}=7.54 \times 10^{-5} \mathrm{eV}^{2}$ and $\Delta m^{2}=2.43 \times 10^{-3} \mathrm{eV}^{2}$ ), one can figure out the neutrino mass spectrum

$$
\begin{aligned}
& m_{3} \approx \sqrt{\Delta m^{2}}=4.9 \times 10^{-2} \mathrm{eV}, \\
& m_{2} \approx m_{3} \sin \theta_{13} \tan \theta_{23} \cot \theta_{12}=9.0 \times 10^{-3} \mathrm{eV}, \\
& m_{1} \approx m_{3} \sin \theta_{13} \tan \theta_{23} \tan \theta_{12}=4.0 \times 10^{-3} \mathrm{eV} .
\end{aligned}
$$

As shown in Eq. (20), there exists an interesting correlation between three mixing angles and the neutrino mass ratios

$$
R_{\nu}=\frac{\delta m^{2}}{\Delta m^{2}} \approx \frac{4 \tan ^{2} \theta_{23} \sin ^{2} \theta_{13}}{\sin 2 \theta_{12} \tan 2 \theta_{12}} .
$$

Taking the best-fit values of three mixing angles, we can get $R_{\nu} \approx 0.027$ from Eq. (33). On the other hand, the best-fit values of two neutrino mass-squared 
differences yield $R_{\nu} \approx 0.031$. Therefore, Pattern $\mathbf{A}_{1}$ is well consistent with current oscillation data, and will be soon tested in the future neutrino oscillation experiments. In the case of $\delta=\pi$, we can obtain

$$
\begin{aligned}
& \xi=-\eta \cdot \frac{s_{13}}{c_{13}^{2}}\left(\frac{s_{12} s_{23}}{c_{12} c_{23}}+s_{13}\right), \\
& \zeta=+\chi \cdot \frac{s_{13}}{c_{13}^{2}}\left(\frac{c_{12} s_{23}}{s_{12} c_{23}}-s_{13}\right),
\end{aligned}
$$

where $\eta=-1$ and $\chi=+1$ are implied. To the leading order of $s_{13}$, the phenomenological implications in the case of $\delta=\pi$ are the same as those in the case of $\delta=0$, so we shall not discuss this case further. More precise measurements of neutrino mixing angles are needed to distinguish between these two cases.

- Pattern $\mathbf{A}_{2}$ with $\left(M_{\nu}\right)_{e e}=\left(M_{\nu}\right)_{e \tau}=0$. All the analytical results of Pattern $\mathbf{A}_{\mathbf{2}}$ can be obtained from those of Pattern $\mathbf{A}_{\mathbf{1}}$ with the replacements $\theta_{23} \rightarrow \pi / 2-\theta_{23}$ and $\delta \rightarrow \delta-\pi$. Therefore, in the case of $\delta=0$, we have

$$
\begin{aligned}
& \xi=-\eta \cdot \frac{s_{13}}{c_{13}^{2}}\left(\frac{s_{12} c_{23}}{c_{12} s_{23}}+s_{13}\right), \\
& \zeta=+\chi \cdot \frac{s_{13}}{c_{13}^{2}}\left(\frac{c_{12} c_{23}}{s_{12} s_{23}}-s_{13}\right) .
\end{aligned}
$$

After taking $\eta=-1$ and $\chi=+1$ and neglecting the terms of $\mathcal{O}\left(s_{13}^{2}\right)$, one can obtain

$$
\begin{aligned}
& \xi \approx \sin \theta_{13} \cot \theta_{23} \tan \theta_{12}, \\
& \zeta \approx \sin \theta_{13} \cot \theta_{23} \cot \theta_{12} .
\end{aligned}
$$

Given $0.59 \leq \tan \theta_{12} \leq 0.75,0.70 \leq \tan \theta_{23} \leq 1.3$, and $0.13 \leq \sin \theta_{13} \leq 0.18$ at the $3 \sigma$ level, we can verify that $\xi<\zeta<1$, implying that only the normal neutrino mass hierarchy is allowed. The neutrino mass spectrum turns out to be

$$
\begin{aligned}
& m_{3} \approx \sqrt{\Delta m^{2}}, \\
& m_{2} \approx m_{3} \sin \theta_{13} \cot \theta_{23} \tan \theta_{12}, \\
& m_{1} \approx m_{3} \sin \theta_{13} \cot \theta_{23} \cot \theta_{12} .
\end{aligned}
$$

As in the case of Pattern $\mathbf{A}_{\mathbf{1}}$, there is a constraint relation among three mixing angles and neutrino mass ratios. To the leading order, we get

$$
R_{\nu}=\frac{\delta m^{2}}{\Delta m^{2}} \approx \frac{4 \cot ^{2} \theta_{23} \sin ^{2} \theta_{13}}{\sin 2 \theta_{12} \tan 2 \theta_{12}}
$$

Although Eq. (38) is not fulfilled by the best-fit values of $\left(\theta_{12}, \theta_{23}, \theta_{13}\right)$ and $\left(\delta m^{2}, \Delta m^{2}\right)$, Pattern $\mathbf{A}_{\mathbf{2}}$ is indeed compatible with current oscillation data at the $3 \sigma$ level, as will be demonstrated by the numerical analysis in subsection 3.2 . In a similar way, one can discuss the case of $\delta=\pi$, for which the analytical results can be obtained from Eqs. (30) and (31) by replacing $\theta_{23}$ with $\pi / 2-\theta_{23}$. 
- Pattern $\mathbf{B}_{1}$ with $\left(M_{\nu}\right)_{\mu \mu}=\left(M_{\nu}\right)_{e \tau}=0$. With the help of Eq. (16), we obtain

$$
\begin{aligned}
& \xi=\eta \cdot \frac{-s_{12} c_{12} s_{23}^{3} c_{13}^{2} \mp\left(c_{12}^{2} c_{23}^{2}+s_{12}^{2} s_{23}^{2}\right) c_{23} s_{13}+2 s_{12} c_{12} s_{23} c_{23}^{2} s_{13}^{2}}{s_{12} c_{12} s_{23} c_{23}^{2} \pm\left(s_{12}^{2}-c_{12}^{2}\right) c_{23}^{3} s_{13}+s_{12} c_{12} s_{23} s_{13}^{2}\left(1+c_{23}^{2}\right)}, \\
& \zeta=\chi \cdot \frac{-s_{12} c_{12} s_{23}^{3} c_{13}^{2} \pm\left(c_{12}^{2} c_{23}^{2}+s_{12}^{2} s_{23}^{2}\right) c_{23} s_{13}+2 s_{12} c_{12} s_{23} c_{23}^{2} s_{13}^{2}}{s_{12} c_{12} s_{23} c_{23}^{2} \pm\left(s_{12}^{2}-c_{12}^{2}\right) c_{23}^{3} s_{13}+s_{12} c_{12} s_{23} s_{13}^{2}\left(1+c_{23}^{2}\right)}
\end{aligned}
$$

where the upper and lower signs refer to the cases of $\delta=0$ and $\delta=\pi$, respectively. In the leading order approximation, one can get $\eta=\chi=-1$ and $\xi \approx \zeta \approx \tan ^{2} \theta_{23}$. In the next-to-leading order approximation, we find

$$
\xi-\zeta \approx \pm \frac{4 \sin \theta_{13}}{\sin 2 \theta_{12} \sin 2 \theta_{23}} .
$$

Since $\delta m^{2}>0$ or equivalently $\zeta>\xi$, only $\delta=\pi$ is allowed. In this case, the constraint relation turns out to be

$$
R_{\nu} \approx \frac{2 \sin \theta_{13}}{\sin 2 \theta_{12}}\left|\tan 2 \theta_{23}\right| \text {. }
$$

Note that $R_{\nu} \propto \sin \theta_{13}$ in Eq. (41) may be one order of magnitude larger than $R_{\nu} \propto \sin ^{2} \theta_{13}$ in Eq. (33) or Eq. (38). Taking the values of $\left(\theta_{12}, \theta_{23}, \theta_{13}\right)$ in the $3 \sigma$ ranges, one verify that $R_{\nu}>0.8$, which is obviously in conflict with the experimental observation $R_{\nu}<0.038$. Therefore, we conclude that Pattern $\mathbf{B}_{1}$ has already been excluded by current neutrino oscillation data. Due to the permutation symmetry between Pattern $\mathbf{B}_{\mathbf{1}}$ and Pattern $\mathbf{B}_{\mathbf{2}}$, Eq. (41) is also applicable to the latter, implying that Pattern $\mathbf{B}_{\mathbf{2}}$ has been experimentally ruled out as well.

- Pattern $\mathbf{B}_{3}$ with $\left(M_{\nu}\right)_{\mu \mu}=\left(M_{\nu}\right)_{e \mu}=0$. With the help of Eq. (16), we obtain

$$
\begin{aligned}
& \xi=-\eta \cdot \frac{s_{23}}{c_{23}} \cdot \frac{s_{12} s_{23} \mp c_{12} c_{23} s_{13}}{s_{12} c_{23} \pm c_{12} s_{23} s_{13}}, \\
& \zeta=-\chi \cdot \frac{s_{23}}{c_{23}} \cdot \frac{c_{12} s_{23} \pm s_{12} c_{23} s_{13}}{c_{12} c_{23} \mp s_{12} s_{23} s_{13}},
\end{aligned}
$$

where the upper and lower signs refer to the cases of $\delta=0$ and $\delta=\pi$, respectively. In the leading order approximation, $\eta=\chi=-1$ and $\xi \approx \zeta \approx \tan ^{2} \theta_{23}$ hold, as for Pattern $\mathbf{B}_{\mathbf{1}}$. However, in the next-to-leading order approximation, one gets

$$
\xi-\zeta \approx \mp \frac{4 \tan ^{2} \theta_{23} \sin \theta_{13}}{\sin 2 \theta_{12} \sin 2 \theta_{23}}
$$

and

$$
R_{\nu} \approx \frac{2 \sin ^{2} \theta_{13}}{\sin 2 \theta_{12}} \tan ^{2} \theta_{23}\left|\tan 2 \theta_{23}\right|
$$

It is evident from Eq. (43) that only $\delta=0$ is allowed, because of $\zeta>\xi$. By taking the values of $\left(\theta_{12}, \theta_{23}, \theta_{13}\right)$ in the $3 \sigma$ ranges, we can obtain $R_{\nu}>0.4$, which is far outside of the $3 \sigma$ range of $R_{\nu}$. Hence Pattern $\mathbf{B}_{\mathbf{3}}$ is not compatible with 
current neutrino oscillation data, nor is Pattern $\mathbf{B}_{\mathbf{4}}$ due to the permutation symmetry.

- Pattern C with $\left(M_{\nu}\right)_{\mu \mu}=\left(M_{\nu}\right)_{\tau \tau}=0$. With the help of Eq. (16), we obtain

$$
\begin{aligned}
& \xi=\eta \cdot \frac{c_{12} c_{13}^{2}}{s_{13}} \cdot \frac{c_{12}\left(c_{23}^{2}-s_{23}^{2}\right)-2 s_{12} s_{23} c_{23} s_{13} c_{\delta}}{2 s_{12} c_{12} s_{23} c_{23} c_{\delta}\left(1+s_{13}^{2}\right)-\left(c_{12}^{2}-s_{12}^{2}\right)\left(c_{23}^{2}-s_{23}^{2}\right) s_{13}}, \\
& \zeta=\chi \cdot \frac{s_{12} c_{13}^{2}}{s_{13}} \cdot \frac{s_{12}\left(s_{23}^{2}-c_{23}^{2}\right)-2 c_{12} s_{23} c_{23} s_{13} c_{\delta}}{2 s_{12} c_{12} s_{23} c_{23} c_{\delta}\left(1+s_{13}^{2}\right)-\left(c_{12}^{2}-s_{12}^{2}\right)\left(c_{23}^{2}-s_{23}^{2}\right) s_{13}},
\end{aligned}
$$

where we have defined $c_{\delta}=\cos \delta$. Note that the leptonic $\mathrm{CP}$ violation is allowed in this case. Generally speaking, it is inappropriate to expand Eq. (45) in terms of $s_{13}$ and ignore the higher-order terms, because $c_{\delta}$ and $c_{23}^{2}-s_{23}^{2}$ could be vanishingly small according to current neutrino oscillation data. But the analytical approximations in some interesting limits deserve further discussions:

(1) $\delta=\pi / 2$ and $\theta_{23} \neq \pi / 4$. Insetting $\delta=\pi / 2$ into Eq. (45), one arrives at $\xi=-\eta \cot ^{2} \theta_{13} \cos ^{2} \theta_{12} / \cos 2 \theta_{12}$ and $\zeta=+\chi \cot ^{2} \theta_{13} \sin ^{2} \theta_{12} / \cos 2 \theta_{12}$, which are independent of $\theta_{23}$. Since $\cos ^{2} \theta_{12}>\sin ^{2} \theta_{12}$ holds, we are led to $\eta=-1, \chi=+1$, and $\xi>\zeta$. This observation indicates that the maximal $\mathrm{CP}$-violating phase is not allowed if $\theta_{23} \neq \pi / 4$.

(2) $\delta \neq \pi / 2$ and $\theta_{23}=\pi / 4$. Assuming $\theta_{23}=\pi / 4$ in Eq. (45), we can get $\eta=\chi=-1$ and $\xi=\zeta=\cos ^{2} \theta_{13} /\left(1+\sin ^{2} \theta_{13}\right)<1$, which only depends on $\theta_{13}$. Hence the maximal mixing $\theta_{23}=\pi / 4$ is not favored if $\delta \neq \pi / 2$, and a tiny deviation of $\theta_{23}$ from $\pi / 4$ is necessary to break the degeneracy between $m_{1}$ and $m_{2}$.

(3) $\delta=\pi / 2$ and $\theta_{23}=\pi / 4$. In this case, one can verify that $\left|U_{\mu i}\right|^{2}=\left|U_{\tau i}\right|^{2}$ (for $i=1,2,3$ ) hold, so Eq. (16) is not applicable. The equality $\left(M_{\nu}\right)_{\mu \mu}=$ $\left(M_{\nu}\right)_{\tau \tau}$ implies that only one constraint relation is obtained by requiring $\left(M_{\nu}\right)_{\mu \mu}=0$. Assuming $\delta=\pi / 2$ and $\theta_{23}=\pi / 4$, we find

$$
\xi\left(c_{12}^{2} s_{13}^{2}+s_{12}^{2}\right)+\zeta\left(s_{12}^{2} s_{13}^{2}+c_{12}^{2}\right)=c_{13}^{2}
$$

in the case of $\eta=\chi=-1$. If the higher-order terms of $\mathcal{O}\left(s_{13}^{2}\right)$ are neglected, then one obtains $\xi=\left(1-\zeta \cdot c_{12}^{2}\right) / s_{12}^{2}$, implying that neither $\zeta>\xi>1$ nor $1>\zeta>\xi$ is allowed. Consequently, both $\xi$ and $\zeta$ should be close to one, and the deviations from one are of $\mathcal{O}\left(s_{13}^{2}\right)$. Based on this observation, we define $\xi \equiv 1-\Delta \xi$ and $\zeta \equiv 1-\Delta \zeta$, and then solve Eqs. (20) and (46) for $\Delta \xi$ and $\Delta \zeta$. It is straightforward to get

$$
\begin{aligned}
& \Delta \xi=\frac{\left(2+R_{\nu}\right) \sin ^{2} \theta_{13}}{1-R_{\nu} \cos 2 \theta_{12} / 2}, \\
& \Delta \zeta=\frac{\left(2-R_{\nu}\right) \sin ^{2} \theta_{13}}{1-R_{\nu} \cos 2 \theta_{12} / 2} .
\end{aligned}
$$

Hence the neutrino masses are nearly degenerate, while both $\delta \approx \pi / 2$ and $\theta_{23} \approx \pi / 4$ are allowed and expected to be valid simultaneously. 
(4) $\delta \neq \pi / 2$ and $\theta_{23} \neq \pi / 4$. Furthermore, we assume $c_{\delta} \gg s_{13}$ and then obtain

$$
\begin{aligned}
& \xi \approx-\eta \cdot\left(1-\frac{\cot \theta_{12} \cot 2 \theta_{23}}{\sin \theta_{13} \cos \delta}\right), \\
& \zeta \approx-\chi \cdot\left(1+\frac{\tan \theta_{12} \cot 2 \theta_{23}}{\sin \theta_{13} \cos \delta}\right) .
\end{aligned}
$$

If $\cot 2 \theta_{23} \cos \delta>0$ holds, one can set $\chi=-1$ and then obtain $\zeta>1$, indicating the inverted mass hierarchy $m_{2}>m_{1}>m_{3}$. In this case, $\xi$ is required to be larger than one but smaller than $\zeta$, and this can be achieved by setting $\eta=+1$ and $\cot \theta_{12} \cot 2 \theta_{23}>2 \sin \theta_{13} \cos \delta>2 \cot 2 \theta_{12} \cot 2 \theta_{23}$. It is straightforward to verify that $\cot 2 \theta_{23} \cos \delta \leq 0$ contradicts with the requirement $\zeta>\xi$. Therefore, only the inverted mass hierarchy is allowed.

From the above discussions, one can observe that there exists a small parameter space around $\delta=\pi / 2$ and $\theta_{23}=\pi / 4$, where the normal mass hierarchy is allowed and neutrino masses are in fact nearly degenerate. In the main part of parameter space, only the inverted mass hierarchy is consistent with current oscillation data. For the general case, the numerical analysis will be done in subsection 3.2.

- Pattern $\mathbf{D}_{1}$ with $\left(M_{\nu}\right)_{\mu \mu}=\left(M_{\nu}\right)_{\mu \tau}=0$. With the help of Eq. (16), we obtain

$$
\begin{aligned}
& \xi \approx \cot \theta_{12} \tan \theta_{23}, \\
& \zeta \approx \tan \theta_{12} \tan \theta_{23},
\end{aligned}
$$

where $\eta=-1$ and $\chi=+1$ are taken, and the higher-order terms $\mathcal{O}\left(s_{13}^{2}\right)$ have been safely neglected. Together with the oscillation data, Eq. (49) indicates $\zeta<\xi$, which is in contradiction with the experimental observation $m_{2}>m_{1}$. Therefore, this pattern is not viable, nor is Pattern $\mathbf{D}_{\mathbf{2}}$ due to the permutation symmetry.

- Pattern $\mathbf{E}_{1}$ with $\left(M_{\nu}\right)_{e e}=\left(M_{\nu}\right)_{\mu \mu}=0$. With the help of Eq. (16), we obtain

$$
\begin{aligned}
& \xi \approx \sin ^{2} \theta_{12} \tan ^{2} \theta_{23} / \cos 2 \theta_{12}, \\
& \zeta \approx \cos ^{2} \theta_{12} \tan ^{2} \theta_{23} / \cos 2 \theta_{12},
\end{aligned}
$$

in the leading-order approximation, where we have set $\eta=+1$ and $\chi=-1$. Since the neutrino mass ratios have been determined as in Eq. (50), one can get

$$
R_{\nu} \approx \frac{4 \tan ^{4} \theta_{23} \cos 2 \theta_{12}}{\left|\tan ^{4} \theta_{23}\left(1+\cos ^{2} 2 \theta_{12}\right)-4 \cos ^{2} 2 \theta_{12}\right|},
$$

implying $R_{\nu}>2 \cos 2 \theta_{12}$ or $R_{\nu}>\tan ^{4} \theta_{23} / \cos 2 \theta_{12}$. Taking the allowed values of $\theta_{23}$ and $\theta_{12}$ in the $3 \sigma$ ranges, we can derive $R_{\nu}>0.56$ or $R_{\nu}>0.50$, respectively. Therefore, Pattern $\mathbf{E}_{1}$ is not consistent with current neutrino oscillation data, nor is Pattern $\mathbf{E}_{\mathbf{2}}$ according to the permutation symmetry.

- Pattern $\mathbf{E}_{3}$ with $\left(M_{\nu}\right)_{e e}=\left(M_{\nu}\right)_{\mu \tau}=0$. With the help of Eq. (16), we obtain

$$
\begin{aligned}
& \xi \approx \sin ^{2} \theta_{12} / \cos 2 \theta_{12}, \\
& \zeta \approx \cos ^{2} \theta_{12} / \cos 2 \theta_{12},
\end{aligned}
$$


in the leading-order approximation, where we have chosen $\eta=-1$ and $\chi=+1$. The ratio of neutrino mass-squared differences turns out to be

$$
R_{\nu} \approx \frac{4 \cos 2 \theta_{12}}{1-3 \cos ^{2} 2 \theta_{12}}>4 \cos 2 \theta_{12},
$$

which is obviously disfavored by current experimental data.

In summary, we conclude that only three two-zero patterns (i.e., $\mathbf{A}_{\mathbf{1}}, \mathbf{A}_{\mathbf{2}}$ and C) are consistent with current neutrino oscillation data. The detailed numerical analysis of these three patterns will be performed in the following subsection.

\subsection{Numerical Analysis}

We have numerically confirmed that only three patterns $\mathbf{A}_{1}, \mathbf{A}_{\mathbf{2}}$ and $\mathbf{C}$ are viable, as obtained from the above analytical approximations. Our strategy for numerical calculations is as follows:

(1) For each of the twelve patterns of $M_{\nu}$ in Eqs. (3)-(7) we generate a set of random numbers of $\left(\theta_{12}, \theta_{23}, \theta_{13}, \delta m^{2}\right)$ lying in their $3 \sigma$ ranges, which are already given in Eqs. (22)-(25). For the CP-conserving patterns, we consider both cases of $\delta=0$ and $\delta=\pi$. For the CP-violating patterns, $\delta$ is allowed to vary in the range $[0,2 \pi)$.

(2) With the above generated numbers, we can calculate the other physical parameters of $M_{\nu}$. First of all, the neutrino mass ratios $\xi$ and $\zeta$ can be determined through three mixing angles and the CP-violating phase. To be consistent with the experimental data, the following two conditions should be satisfied: (a) $m_{2}>m_{1}$ or equivalently $\zeta^{2}>\xi^{2}$; (b) since only the neutrino mass hierarchies $m_{2}>m_{1}>m_{3}$ and $m_{3}>m_{2}>m_{1}$ are allowed, we further require $\left(\zeta^{2}-1\right)\left(\xi^{2}-1\right)>0$. With the calculated neutrino mass ratios $\xi$ and $\zeta$, one can figure out the absolute neutrino masses $m_{i}$ (for $i=1,2,3$ ) by inserting the randomly generated $\delta m^{2}$ into Eq. (21). Finally we compute $\Delta m^{2}$ via neutrino masses $m_{i}$, the patterns can be regarded as viable if the computed $\Delta m^{2}$ is consistent with the experimental values in Eqs. (23) and (25).

(3) From all the points satisfying the above consistency conditions, we can figure out three neutrino mass eigenvalues $\left(m_{1}, m_{2}, m_{3}\right)$ via Eq. (21). For the $\mathrm{CP}$-violating patterns, it is possible to calculate the Jarlskog invariant $\mathcal{J} \equiv$ $s_{12} c_{12} s_{23} c_{23} s_{13} c_{13}^{2} s_{\delta}$. To present numerical results, we show the allowed regions of three neutrino mass eigenvalues $\left(m_{1}, m_{2}, m_{3}\right)$, those of three mixing angles $\left(\theta_{12}, \theta_{23}, \theta_{13}\right)$, and those of $(\mathcal{J}, \delta)$ if $\mathrm{CP}$ violation is allowed.

Our numerical results are shown in Figs. 1-3. Some comments and discussions are in order:

- Pattern $\mathbf{A}_{1}-$ Our numerical results show that the patterns $\mathbf{A}_{1}$ and $\mathbf{A}_{2}$ survive current experimental tests, which confirms our analytical calculations. The allowed ranges of neutrino mass eigenvalues and mixing angles for Pattern $\mathbf{A}_{\mathbf{1}}$ 
and Pattern $\mathbf{A}_{\mathbf{2}}$ are depicted in Fig. 1 and Fig. 2, respectively. Since the patterns $\mathbf{A}_{\mathbf{1}}$ and $\mathbf{A}_{\mathbf{2}}$ are similar to each other due to the permutation symmetry, here we focus on the former. From Fig. 1, some interesting observations should be noted: (a) Only the normal mass hierarchy $m_{1}<m_{2}<m_{3}$ is allowed. (b) The deviation of $\theta_{23}$ from $\pi / 4$ is expected in both cases of $\delta=0$ and $\delta=\pi$. However, $\theta_{23}<45^{\circ}$ is favored for $\delta=0$, while $\theta_{23}>45^{\circ}$ for $\delta=\pi$. (c) The parameter space in the case of $\delta=0$ receives more stringent constraints than that in the case of $\delta=\pi$. Therefore, more precise measurements of neutrino mixing angles are required to distinguish between the cases of $\delta=0$ and $\delta=\pi$, as well as between Pattern $\mathbf{A}_{\mathbf{1}}$ and Pattern $\mathbf{A}_{\mathbf{2}}$.

- Pattern C- Unlike the previous two patterns, the leptonic CP violation is allowed for this pattern. Our numerical results are given in Fig. 3, where only the inverted mass hierarchy has been considered. From the analytical analysis in section 3.1, the normal mass hierarchy is indeed allowed, if $\theta_{23} \approx \pi / 4$ and $\delta \approx \pi / 2$. However, the main parameter space points to the inverted neutrino mass hierarchy. Although the mixing angles for this pattern are not strictly constrained, the discovery of $\mathrm{CP}$ violation in the future long-baseline neutrino oscillation experiments will definitely single out Pattern $\mathbf{C}$ among all the twozero textures as the true Dirac neutrino mass matrix.

Finally it is worth pointing out that we have performed a numerical analysis of the two-zero textures of $M_{\nu}$ by the oscillation data at the $2 \sigma$ level. We find that all these three patterns are compatible with current experimental data at this level, although the corresponding parameter space is somewhat smaller.

\section{One-zero Textures}

Now we proceed to consider the one-zero textures of Dirac neutrino mass matrix $M_{\nu}$. Since only one zero element is assumed, these textures are not as predictive as the two-zero ones. On the other hand, it will be no doubt that all the six patterns in Eqs. (9) and (10) are compatible with current experimental data, which has also been confirmed by numerical calculations. For simplicity, we focus on the analytical analysis by making some reasonable approximations.

- Pattern $\mathbf{P}_{1}$ with $\left(M_{\nu}\right)_{e e}=0$. With the help of Eq. (12), we get

$$
\eta \cdot \xi c_{12}^{2} c_{13}^{2}+\chi \cdot \zeta s_{12}^{2} c_{13}^{2}+s_{13}^{2}=0 .
$$

Since only one constraint relation exists, it is by no means possible to pin down both the neutrino mass spectrum and the CP-violating phase. From Eq. (54), one can observe that $\delta$ is entirely unconstrained. In this case, however, neutrino mass eigenvalues can be determined, as we shall show below. To find the solutions to Eq. (54), one has to consider three different possibilities: (1) $\eta=\chi=-1$; (2) $\eta=-1$ and $\chi=+1$; (3) $\eta=+1$ and $\chi=-1$. Now we examine whether all these possibilities are allowed by current experimental data. 
(1) If $\eta=\chi=-1$ is assumed, one can observe from Eq. (54) that both $\xi$ and $\zeta$ should be of $\mathcal{O}\left(s_{13}^{2}\right)$, and furthermore obtain

$$
\zeta=\tan ^{2} \theta_{13} / \sin ^{2} \theta_{12}-\xi \cot ^{2} \theta_{12},
$$

from which $\xi<\tan ^{2} \theta_{13}$ can be derived by requiring $\zeta>\xi$. Since both $\xi$ and $\zeta$ are quite small in this case, $R_{\nu} \approx \zeta^{2}-\xi^{2}$ holds as an excellent approximation. To be explicit, one can find

$$
\begin{aligned}
& \xi=\frac{\tan ^{2} \theta_{13} \cot ^{2} \theta_{12}-\sqrt{\tan ^{4} \theta_{13}+R_{\nu} \cos 2 \theta_{12}}}{\cot ^{2} \theta_{12}-1}, \\
& \zeta=\frac{\tan ^{2} \theta_{13} \tan ^{2} \theta_{12}-\sqrt{\tan ^{4} \theta_{13}+R_{\nu} \cos 2 \theta_{12}}}{\tan ^{2} \theta_{12}-1},
\end{aligned}
$$

which together with Eq. (21) leads to the neutrino mass spectrum. In addition, the condition $\tan ^{2} \theta_{13} \geq \sqrt{R_{\nu}} \sin ^{2} \theta_{12}$ has to be fulfilled to guarantee a non-negative $\xi$. Given $0.259 \leq \sin ^{2} \theta_{12} \leq 0.359,0.017 \leq \tan ^{2} \theta_{13} \leq 0.032$, and $0.027 \leq R_{\nu} \leq 0.037$ at the $3 \sigma$ level for the normal neutrino mass hierarchy, it is straightforward to verify that such a condition cannot be satisfied. Hence there is no solution in this case.

(2) If $\eta=+1$ and $\chi=-1$ are taken, then Eq. (54) can be written as

$$
\zeta=\tan ^{2} \theta_{13} / \sin ^{2} \theta_{12}+\xi \cot ^{2} \theta_{12} .
$$

Different from the previous case, both $\zeta$ and $\xi$ need not to be as small as $\tan ^{2} \theta_{13}$. Requiring $\zeta>\xi$ leads to $\cot ^{2} \theta_{12}>1$, which is favored by current oscillation data. Inserting Eq. (57) into Eq. (20), we can figure out the neutrino mass ratios

$$
\begin{aligned}
& \xi=\frac{\tan ^{2} \theta_{13} \cot ^{2} \theta_{12}-\sqrt{\tan ^{4} \theta_{13}+R_{\nu} \cos 2 \theta_{12}}}{1-\cot ^{2} \theta_{12}}, \\
& \zeta=\frac{\tan ^{2} \theta_{13} \tan ^{2} \theta_{12}+\sqrt{\tan ^{4} \theta_{13}+R_{\nu} \cos 2 \theta_{12}}}{1-\tan ^{2} \theta_{12}},
\end{aligned}
$$

where we have assumed $\xi^{2}<\zeta^{2} \ll 1$, and thus $R_{\nu} \approx \zeta^{2}-\xi^{2}$. For the best-fit values $\theta_{12}=33.6^{\circ}, \theta_{13}=8.9^{\circ}$, and $R_{\nu}=0.031$, the neutrino mass ratios are given as $\xi=0.045$ and $\zeta=0.22$, which justifies the assumption of $\xi^{2}<\zeta^{2} \ll 1$. With the help of Eq. (21), the neutrino mass eigenvalues are found to be $m_{3} \approx \sqrt{\delta m^{2} /\left(\zeta^{2}-\xi^{2}\right)}=0.04 \mathrm{eV}, m_{2}=\zeta m_{3} \approx 8.8 \times 10^{-3} \mathrm{eV}$ and $m_{1}=\xi m_{3} \approx 1.8 \times 10^{-3} \mathrm{eV}$.

(3) If $\eta=-1$ and $\chi=+1$ are taken, then Eq. (54) turns out to be

$$
\zeta=\xi \cot ^{2} \theta_{12}-\tan ^{2} \theta_{13} / \sin ^{2} \theta_{12} .
$$

The requirement $\zeta>\xi$ gives rise to a lower bound $\xi>\tan ^{2} \theta_{13} / \cos 2 \theta_{12}$. In a similar way to the previous case, we assume $\xi^{2}<\zeta \ll 1$ and thus 
$R_{\nu} \approx \zeta^{2}-\xi^{2}$. Consequently, we arrive at

$$
\begin{aligned}
& \xi=\frac{\tan ^{2} \theta_{13}+\tan ^{2} \theta_{12} \sqrt{\tan ^{4} \theta_{13}+R_{\nu} \cos 2 \theta_{12}}}{1-\tan ^{2} \theta_{12}}, \\
& \zeta=\frac{\tan ^{2} \theta_{13} \tan ^{2} \theta_{12}+\sqrt{\tan ^{4} \theta_{13}+R_{\nu} \cos 2 \theta_{12}}}{1-\tan ^{2} \theta_{12}} .
\end{aligned}
$$

For the best-fit values $\theta_{12}=33.6^{\circ}, \theta_{13}=8.9^{\circ}$, and $R_{\nu}=0.031$, the neutrino mass ratios are given as $\xi=0.13$ and $\zeta=0.22$, which justifies the assumption of $\xi^{2}<\zeta^{2} \ll 1$. The neutrino masses turn out to be $m_{3}=\sqrt{\delta m^{2} /\left(\zeta^{2}-\xi^{2}\right)} \approx 0.049 \mathrm{eV}, m_{2}=\zeta m_{3} \approx 0.01 \mathrm{eV}$, and $m_{1}=$ $\xi m_{3} \approx 6.4 \times 10^{-3} \mathrm{eV}$.

So we conclude that Pattern $\mathbf{P}_{\mathbf{1}}$ is consistent with current neutrino oscillation data, and only the normal mass hierarchy is allowed. Additionally, the CPviolating phase $\delta$ is arbitrary.

- Pattern $\mathbf{P}_{2}$ with $\left(M_{\nu}\right)_{\mu \mu}=0$. With the help of Eq. (12), we get

$\eta \cdot \xi\left(s_{12}^{2} c_{23}^{2}+2 \mathcal{J} t_{\delta}^{-1} c_{13}^{-2}+c_{12}^{2} s_{23}^{2} s_{13}^{2}\right)+\chi \cdot \zeta\left(c_{12}^{2} c_{23}^{2}-2 \mathcal{J} t_{\delta}^{-1} c_{13}^{-2}+s_{12}^{2} s_{23}^{2} s_{13}^{2}\right)+s_{23}^{2} c_{13}^{2}=0$,

where $\mathcal{J} \equiv s_{12} c_{12} s_{23} c_{23} s_{13} c_{13}^{2} s_{\delta}$ is the Jarlskog invariant in the standard parametrization, and $t_{\delta} \equiv \tan \delta$ has been defined. For simplicity, we assume $\eta=\chi=-1$ and solve Eq. (61) for $\xi$ and $\zeta$ in the leading-order approximation. In this case, it is straightforward to observe $\xi \approx \zeta \approx \tan ^{2} \theta_{23}$. In order to figure out the deviations of $\xi$ and $\zeta$ from $\tan ^{2} \theta_{23}$, we define $\xi \equiv \tan ^{2} \theta_{23}-\Delta \xi$ and $\zeta \equiv \tan ^{2} \theta_{23}-\Delta \zeta$, and insert them back into Eq. (61). Then one can see that both $\Delta \xi$ and $\Delta \zeta$ should be of $\mathcal{O}\left(s_{13}^{2}\right)$. Combining Eq. (61) with Eq. (20), we obtain

$$
\Delta \xi \approx \frac{\left(1-\tan ^{4} \theta_{23}\right) R_{\nu}+2 \tan ^{4} \theta_{23} \sin ^{2} \theta_{13} /\left(\cos ^{2} \theta_{12} \cos ^{2} \theta_{23}\right)}{2 \tan ^{2} \theta_{23} / \cos ^{2} \theta_{12}-\tan ^{2} \theta_{23}\left(1-\tan ^{2} \theta_{12}\right) R_{\nu}},
$$

and $\Delta \zeta=\sin ^{2} \theta_{13} \tan ^{2} \theta_{23} /\left(\cos ^{2} \theta_{12} \cos ^{2} \theta_{23}\right)-\Delta \xi \tan ^{2} \theta_{12}$, where we have assumed the normal mass hierarchy. Taking the best-fit values of $\left(\theta_{12}, \theta_{23}, \theta_{13}\right)$ and $\left(\delta m^{2}, \Delta m^{2}\right)$ for example, one can get $\xi=0.59$ and $\zeta=0.61$, and the neutrino masses are $m_{3}=6.4 \times 10^{-2} \mathrm{eV}, m_{2}=3.9 \times 10^{-2} \mathrm{eV}$ and $m_{1}=3.8 \times 10^{-2} \mathrm{eV}$. Whether the neutrino mass hierarchy is normal or inverted depends on the mixing angle $\theta_{23}$. Similarly, one can also analyze the case with $\eta \cdot \chi=-1$. It is worthwhile to point out that $\delta$ is almost irrelevant to the determination of neutrino masses, because its contribution to Eq. (61) is suppressed by $\sin \theta_{13}$. Therefore, $\delta$ is totally arbitrary, as for Pattern $\mathbf{P}_{\mathbf{1}}$.

Because of the permutation symmetry, the analytical results for Pattern $\mathbf{P}_{\mathbf{3}}$ can be obtained by the replacements $\theta_{23} \rightarrow \pi / 2-\theta_{23}$ and $\delta \rightarrow \pi-\delta$. Thus we shall not discuss further about this case.

- Pattern $\mathbf{P}_{4}$ with $\left(M_{\nu}\right)_{e \mu}=0$. With the help of Eq. (12), we get

$$
-\eta \cdot \xi c_{12}\left(s_{12} c_{23} \pm c_{12} s_{23} s_{13}\right)+\chi \cdot \zeta s_{12}\left(c_{12} c_{23} \mp s_{12} s_{23} s_{13}\right)+s_{13} s_{23}=0,
$$


where the upper and lower sign stands for $\delta=0$ and $\delta=\pi$, respectively. At the leading order, Eq. (63) approximates to $(\eta \xi-\chi \zeta)=2 \sin \theta_{13} \tan \theta_{23} / \sin 2 \theta_{12}$, implying $\chi=-1$ and $\eta= \pm 1$. Take $\chi=-1$ and $\eta=+1$ for example. Since $\xi+\zeta=2 \sin \theta_{13} \tan \theta_{23} / \sin 2 \theta_{12}$, we see that $R_{\nu} \approx \zeta^{2}-\xi^{2}$ holds as an excellent approximation. Consequently, one gets

$$
\begin{aligned}
& \xi=\frac{4 \tan ^{2} \theta_{23} \sin ^{2} \theta_{13}-R_{\nu} \sin ^{2} 2 \theta_{12}}{4 \sin 2 \theta_{12} \tan \theta_{23} \sin \theta_{13}}, \\
& \zeta=\frac{4 \tan ^{2} \theta_{23} \sin ^{2} \theta_{13}+R_{\nu} \sin ^{2} 2 \theta_{12}}{4 \sin 2 \theta_{12} \tan \theta_{23} \sin \theta_{13}},
\end{aligned}
$$

implying the normal neutrino mass hierarchy. Taking the best-fit values of $\left(\theta_{12}, \theta_{23}, \theta_{13}\right)$ and $\left(\delta m^{2}, \Delta m^{2}\right)$, we have $\xi=0.07$ and $\zeta=0.19$, and thus the neutrino masses $m_{3}=4.9 \times 10^{-2} \mathrm{eV}, m_{2}=9.3 \times 10^{-3} \mathrm{eV}$ and $m_{1}=3.4 \times 10^{-3} \mathrm{eV}$. In the case $\chi=-1$ and $\eta=-1$, it is straightforward to verify that only the inverted mass hierarchy is allowed.

Because of the permutation symmetry, the analytical results for Pattern $\mathbf{P}_{\mathbf{5}}$ can be obtained by the replacements $\theta_{23} \rightarrow \pi / 2-\theta_{23}$ and $\delta \rightarrow \pi-\delta$.

- Pattern $\mathbf{P}_{6}:\left(M_{\nu}\right)_{\mu \tau}=0$, we have

$\eta \xi\left[s_{12}^{2} \mp 2 \cot 2 \theta_{23} c_{12} s_{12} s_{13}-c_{12}^{2} s_{13}^{2}\right]+\chi \zeta\left[c_{12}^{2} \pm 2 \cot 2 \theta_{23} c_{12} s_{12} s_{13}-s_{12}^{2} s_{13}^{2}\right]=c_{1}^{2}(65)$

where the upper and lower sign stands for $\delta=0$ and $\delta=\pi$, respectively. In the leading-order approximation, we can obtain $\eta \xi s_{12}^{2}+\chi \zeta c_{12}^{2}=c_{13}^{2}$. Note that neutrino oscillation data indicate $c_{12}^{2}>s_{12}^{2}$ and $\zeta>\xi$, so only two possibilities need to be considered: (1) $\eta=\chi=+1$; and (2) $\eta=-1$ and $\chi=+1$. Now we examine whether these two possibilities are compatible with current neutrino oscillation data:

(1) If $\eta=\chi=+1$ is assumed, then $\xi \approx \zeta \approx \cos ^{2} \theta_{13}$ holds. In order to figure out the deviations of $\xi$ and $\zeta$ from $\cos ^{2} \theta_{13}$, we define $\xi \equiv \cos ^{2} \theta_{13}-\Delta \xi$ and $\zeta \equiv \cos ^{2} \theta_{13}-\Delta \zeta$, and insert them back into Eq. (65). Then we obtain $\Delta \zeta=-\tan ^{2} \theta_{12} \Delta \xi$. Combing Eq. (65) with Eq. (20), we obtain $\Delta \xi \approx R_{\nu} \sin ^{2} \theta_{13} \cos ^{2} \theta_{12}$. Taking the best-fit values of neutrino mixing parameters, we finally get $m_{3}=0.38 \mathrm{eV}$. Considering $m_{1} \approx m_{2} \approx m_{3}$, we can see this might violate the cosmological limit $\sum m_{i} \equiv m_{1}+m_{2}+$ $m_{3}<0.61 \mathrm{eV}$ from the cosmic microwave background and the large-scale structure observations. ${ }^{78}$

(2) If $\eta=-1$ and $\chi=+1$ are taken, we can get $\xi s_{12}^{2}+1 \approx \zeta c_{12}^{2}$. Current oscillation data indicate $s_{12}^{2}<c_{12}^{2}$, thus $\xi>1$ is required, implying the inverted neutrino mass hierarchy $m_{3}<m_{1}<m_{2}$. Furthermore, combining $\xi \approx \zeta \cot ^{2} \theta_{12}-1 / \sin ^{2} \theta_{12}>1$ with $R_{\nu} \approx 2\left(\zeta^{2}-\xi^{2}\right) /\left(\zeta^{2}+\xi^{2}\right)$, we can obtain $\zeta=1 /\left(\cos ^{2} \theta_{12}-\sin ^{2} \theta_{12} \sqrt{\left(2+R_{\nu}\right) /\left(2-R_{\nu}\right)}\right)$. Taking the best-fit values of neutrino mixing parameters, one can obtain $\zeta=2.62$ and $\xi=2.60$, and the neutrino masses are $m_{3}=2.7 \times 10^{-2} \mathrm{eV}, m_{2}=7.1 \times 10^{-2} \mathrm{eV}$ and $m_{1}=7.0 \times 10^{-2} \mathrm{eV}$. 
Therefore, we conclude that Pattern $\mathbf{P}_{\mathbf{6}}$ is completely consistent with current neutrino oscillation data, and only the inverted mass hierarchy is allowed. However, the absolute neutrino masses might exceed the cosmological bound in the case of a nearly-degenerate neutrino mass spectrum.

\section{Summary}

In light of the recent measurements of $\theta_{13}$ and the latest global-fit analysis of neutrino oscillation experiments, we have performed a systematic study of the Dirac neutrino mass matrix $M_{\nu}$ with two independent texture zeros or one texture zero. It turns out that three two-zero patterns (i.e., $\mathbf{A}_{\mathbf{1 , 2}}$ and $\mathbf{C}$ ) and all six one-zero patterns(i.e., $\mathbf{P}_{\mathbf{1 , 2 , 3 , 4 , 5 , 6}}$ ) can survive current experimental tests at the $3 \sigma$ level. In fact, all of them are found to be compatible with the oscillation data even at the $2 \sigma$ level. Analytical analyses have been done for both two-zero and one-zero textures in order to understand why they are favored or disfavored by current experimental data. Moreover, the allowed parameter space for three viable two-zero textures has been obtained through a detailed numerical analysis. The following is a brief summary of our conclusions:

- In the basis where the charged-lepton mass matrix is diagonal, neutrino masses, flavor mixing angles, and CP-violating phase are determined by the Dirac neutrino mass matrix $M_{\nu}$, which can be further made Hermitian by redefining the right-handed singlet neutrino fields. We demonstrate that one vanishing offdiagonal element in the Hermitian neutrino mass matrix is enough to guarantee $\mathrm{CP}$ conservation in the lepton sector.

- Among fifteen two-zero textures of $M_{\nu}$, only three (i.e., $\mathbf{A}_{\mathbf{1}}, \mathbf{A}_{\mathbf{2}}$ and $\mathbf{C}$ ) turn out to be consistent with current neutrino oscillation data at the $3 \sigma$ level. We have explained in detail why the other patterns are disfavored. For Pattern $\mathbf{A}_{\mathbf{1}}$ and Pattern $\mathbf{A}_{\mathbf{2}}$, only the normal neutrino mass hierarchy is allowed, and there is no $\mathrm{CP}$ violation. For Pattern $\mathbf{C}$, both normal and inverted mass hierarchies are in principle allowed. However, the normal mass hierarchy is possible only if $\delta \approx \pi / 2$ and $\theta_{23} \approx \pi / 4$. The precise measurements of neutrino mixing angles and leptonic $\mathrm{CP}$ violation are needed to distinguish among these currently viable patterns.

- All the six one-zero textures are compatible with current neutrino oscillation data. For the patterns $\mathbf{P}_{\mathbf{4 , 5 , 6}}$, even only one texture zero is assumed, it is possible to fully determine neutrino mass eigenvalues and the $\mathrm{CP}$-violating phase in terms of the observed three mixing angles $\left(\theta_{12}, \theta_{23}, \theta_{13}\right)$ and two neutrino mass-squared differences $\left(\delta m^{2}, \Delta m^{2}\right)$. For the patterns $\mathbf{P}_{\mathbf{1 , 2 , 3}}$, even if one free parameter remains, the full determination of neutrino mass spectrum is possible, at least in the first-order approximation. Although the parameter space for all these patterns has received some constraints, it seems impossible to exclude them experimentally in the near future. 
It is worthwhile to stress that whether neutrinos are Dirac or Majorana particles remains an open question. At present, the only feasible way to demonstrate that neutrinos are Majorana particles is to observe the neutrinoless double-beta decays ${ }^{[79}$ If neutrinos are Dirac particles, such a lepton-number-violating process is forbidden. Therefore, it really makes sense to consider Dirac neutrinos, and to study the flavor mixing and $\mathrm{CP}$ violation in this scenario. The ongoing and upcoming neutrino oscillation experiments are expected to precisely measure the neutrino mixing parameters, in particular the smallest mixing angle $\theta_{13}$, the deviation of $\theta_{23}$ from $\pi / 4$ and the Dirac CP-violating phase $\delta$. The sensitivity of future cosmological observations to the sum of neutrino masses $\sum m_{i}$ will probably reach $\sim 0.05 \mathrm{eV}$ in the near future. We therefore expect that some patterns of the two-zero textures of the neutrino mass matrix $M_{\nu}$ for Dirac neutrinos might be excluded or only marginally allowed by tomorrow's data, and those surviving the experimental tests should shed light on the underlying flavor structure of massive neutrinos.

\section{Acknowledgments}

We are indebted to Prof. Zhi-zhong Xing for suggesting such an investigation and helpful discussions. One of us (X.W.L.) would like to thank Dr. Yu-feng Li for useful discussions, and Theoretical Physics Division of IHEP for financial support and hospitality in Beijing. The main part of this work was done at IHEP, Beijing (X.W.L.) and at Max-Planck-Institut für Physik, München (S.Z.). This work was partially supported by the European Union FP7 INVISIBLES (Marie Curie Actions, PITN-GA-2011-289442) and by the Göran Gustafsson Foundation.

\section{Appendix A. Matrix Polar Decomposition}

In this appendix, we prove that the mass matrix $M_{\nu}$ of Dirac neutrinos can be made Hermitian by redefining the right-handed singlet neutrino fields. In the extension of the standard model with three right-handed singlet neutrinos $\nu_{\mathrm{R}}$, the Lagrangian relevant for lepton masses reads

$$
-\mathcal{L}_{l}=\overline{\ell_{\mathrm{L}}} Y_{l} l_{\mathrm{R}} H+\overline{\ell_{\mathrm{L}}} Y_{\nu} \nu_{\mathrm{R}} \tilde{H}+\text { h.c. },
$$

where $\ell_{\mathrm{L}}$ and $\tilde{H} \equiv i \sigma_{2} H^{*}$ denote the lepton and Higgs doublets, $l_{\mathrm{R}}$ stand for the charged-lepton singlets, $Y_{l}$ and $Y_{\nu}$ are the $3 \times 3$ Yukawa coupling matrices for charged leptons and neutrinos.

Now one can perform the basis transformation $\ell_{\mathrm{L}}^{\prime}=U_{l}^{\dagger} \ell_{\mathrm{L}}$ and $l_{\mathrm{R}}^{\prime}=V_{l}^{\dagger} l_{\mathrm{R}}$ to diagonalize the charged-lepton Yukawa coupling matrix, i.e., $U_{l}^{\dagger} Y_{l} V_{l}=D_{l} \equiv$ $\operatorname{Diag}\left\{y_{e}, y_{\mu}, y_{\tau}\right\}$. In this basis, the neutrino Yukawa coupling matrix turns out to be $Y_{\nu}^{\prime}=U_{l}^{\dagger} Y_{\nu}$. After the electroweak gauge symmetry breaking, the lepton mass term is given by

$$
-\mathcal{L}_{\mathrm{m}}=\overline{l_{\mathrm{L}}} M_{l} l_{\mathrm{R}}+\overline{\nu_{\mathrm{L}}} M_{\nu} \nu_{\mathrm{R}}+\text { h.c. },
$$


where $M_{l}=D_{l} v$ and $M_{\nu}=Y_{\nu}^{\prime} v$ with $v \approx 174 \mathrm{GeV}$ being the vacuum expectation value of the Higgs field. In the chosen basis, $M_{l}=\operatorname{Diag}\left\{m_{e}, m_{\mu}, m_{\tau}\right\}$ is diagonal with the charged-lepton masses $m_{\alpha}=y_{\alpha} v$ (for $\alpha=e, \mu, \tau$ ), while $M_{\nu}$ is in general an arbitrary $3 \times 3$ complex matrix. In the following, we shall show that the neutrino mass matrix can be decomposed as $M_{\nu}=S_{\nu} \cdot V_{\nu}$, where $S_{\nu}$ is an Hermitian matrix and $V_{\nu}$ is a unitary matrix. Therefore, we can redefine the right-handed neutrino field $\nu_{\mathrm{R}}^{\prime}=V_{\nu} \nu_{\mathrm{R}}$ and thus the neutrino mass matrix is Hermitian.

Finally, we prove that an $n \times n$ complex matrix $M$ can be decomposed into $M=S \cdot V$, where $S$ is an $n \times n$ positive semi-definite Hermitian matrix and $V$ is an $n \times n$ unitary matrix. First, we define $H \equiv M M^{\dagger}$, which is obviously an Hermitian matrix with non-negative eigenvalues, so it can be diagonalized by a unitary transformation $U^{\dagger} H U=D^{2} \equiv \operatorname{Diag}\left\{\lambda_{1}^{2}, \lambda_{2}^{2}, \cdots, \lambda_{n}^{2}\right\}$, where $\lambda_{i} \geq 0$ for $i=1,2, \cdots, n$. Then, we choose $S=\sqrt{H}=\sqrt{M M^{\dagger}}$ with $\sqrt{H} \equiv U D U^{\dagger}$, and it is straightforward to verify $S^{\dagger}=S$ and $S^{2}=H$. Note that $S$ is positive semi-definite and Hermitian, and $M, H$ and $S$ have the same rank $m \leq n$. By definition, the orthonormal eigenvectors $\psi_{i}$ (for $i=1,2, \cdots, n$ ) of $S$ are just the column vectors of the unitary matrix $U$, i.e., $U=\left(\psi_{1}, \psi_{2}, \cdots, \psi_{n}\right)$ and $S \psi_{i}=\lambda_{i} \psi_{i}$. Furthermore, we introduce

$$
\phi_{i}=\left\{\begin{array}{cc}
\frac{1}{\lambda_{i}} M^{\dagger} \psi_{i}, & 1 \leq i \leq m \\
\varphi_{i}, & m<i \leq n
\end{array},\right.
$$

where $\left\{\varphi_{m+1}, \varphi_{m+2}, \cdots, \varphi_{n}\right\}$ can be constructed from $\left\{\psi_{m+1}, \psi_{m+2}, \cdots, \psi_{n}\right\}$ such that $\left\{\phi_{i}\right\}$ is a complete orthonormal basis, i.e., $\phi_{i}^{\dagger} \phi_{j}=\delta_{i j}$. Defining an $n \times n$ matrix $V$, whose element is given by $V=\left(\psi_{1}, \psi_{2}, \cdots, \psi_{n}\right)\left(\phi_{1}, \phi_{2}, \cdots, \phi_{n}\right)^{\dagger}$, we can prove that $M$ and $S V$ behave in the same way on the basis vectors $\left\{\psi_{i}\right\}$. The proof is as follows:

(1) Since either $\left\{\phi_{i}\right\}$ or $\left\{\psi_{i}\right\}$ is a complete set of orthonormal vectors, we have two unitary matrices $U=\left(\psi_{1}, \psi_{2}, \cdots, \psi_{n}\right)$ and $U^{\prime}=\left(\phi_{1}, \phi_{2}, \cdots, \phi_{n}\right)$. Thus it is obvious that $V=U U^{\prime \dagger}$ is a unitary matrix.

(2) We show $\psi_{i}^{\dagger} S V=\psi_{i}^{\dagger} M$. More explicitly,

$$
\psi_{i}^{\dagger} S V=\left\{\begin{array}{cc}
\lambda_{i} \psi_{i}^{\dagger} V=\psi_{i}^{\dagger} M, & 1 \leq i \leq m \\
0, & m<i \leq n
\end{array},\right.
$$

where we have used $\psi_{i}^{\dagger} M=\phi_{i}^{\dagger} M=0$ for $m+1 \leq i \leq n$. Since $\left\{\psi_{i}\right\}$ is a complete set of orthonormal basis, we arrive at $M=S \cdot V$.

Although it is always possible to choose $S$ to be positive semi-definite and Hermitian, we have considered the Dirac neutrino mass matrices $M_{\nu}$ to be more general in the sense that the eigenvalues $\lambda_{i}$ can be either positive or negative. 


\section{References}

1. Particle Data Group, J. Beringer et al., Phys. Rev. D 86, 010001 (2012).

2. F.P. An et al. (Daya Bay Collaboration), Phys. Rev. Lett. 108, 171803 (2012).

3. J.K. Ahn et al. (RENO Collaboration), Phys. Rev. Lett. 108, 191802 (2012).

4. S. Weinberg, Trans. New York Acad. Sci. 38, 185 (1977).

5. F. Wilczek and A. Zee, Phys. Lett. 70B, 418 (1977).

6. H. Fritzsch, Phys. Lett. 70B, 436 (1977).

7. H. Fritzsch, Phys. Lett. 73B, 317 (1978).

8. H. Fritzsch, Nucl. Phys. B155, 18 (1979).

9. C.D. Froggatt and H.B. Nielsen, Nucl. Phys. B 147, 277 (1979).

10. H. Fritzsch and Z.Z. Xing, Prog. Part. Nucl. Phys. 45, 1 (2000).

11. Z.Z. Xing, Int. J. Mod. Phys. A 19, 1 (2004).

12. Z.Z. Xing, arXiv:hep-ph/0406049.

13. M. Gupta and G. Ahuja, Int. J. Mod. Phys. A 27, 1230033 (2012).

14. S. Luo and Z.Z. Xing, Int. J. Mod. Phys. A 27, 1230031 (2012).

15. P.H. Frampton, S.L. Glashow, and D. Marfatia, Phys. Lett. B 536, 79 (2002).

16. Z.Z. Xing, Phys. Lett. B 530, 159 (2002).

17. Z.Z. Xing, Phys. Lett. B 539, 85 (2002).

18. W.L. Guo and Z.Z. Xing, Phys. Rev. D 67, 053002 (2003).

19. P.H. Frampton, M.C. Oh, and T. Yoshikawa, Phys. Rev. D 66, 033007 (2002).

20. A. Kageyama, S. Kaneko, N. Shimoyama, and M. Tanimoto, Phys. Lett. B 538, 96 (2002).

21. B.R. Desai, D.P. Roy, and A.R. Vaucher, Mod. Phys. Lett. A 18, 1355 (2003).

22. M. Frigerio and A.Yu. Smirnov, Phys. Rev. D 67, 013007 (2003).

23. M. Honda, S. Kaneko, and M. Tanimoto, JHEP 0309, 028 (2003).

24. G. Bhattacharyya, A. Raychaudhuri, and A. Sil, Phys. Rev. D 67, 073004 (2003).

25. A. Watanabe and K. Yoshioka, JHEP 0605, 044 (2006).

26. R. Mohanta, G. Kranti, and A.K. Giri, hep-ph/0608292.

27. Y. Farzan and A.Yu. Smirnov, JHEP 0701, 059 (2007).

28. S. Dev, S. Kumar, S. Verma, and S. Gupta, Nucl. Phys. B 784, 103 (2007).

29. S. Dev, S. Kumar, S. Verma, and S. Gupta, Phys. Rev. D 76, 013002 (2007).

30. W.L. Guo, Z.Z. Xing, and S. Zhou, Int. Mod. Phys. E 16, 1 (2007).

31. S. Rajpoot, arXiv: hep-ph/0703185

32. H.A. Alhendi, E.I. Lashin, A.A. Mudlei, Phys. Rev. D 77, 013009 (2008).

33. E.I. Lashin and N. Chamoun, Phys. Rev. D 78, 073002 (2008).

34. A. Dighe and N. Sahu, arXiv:0812.0695.

35. S. Goswami and A. Watanabe, Phys. Rev. D 79, 033004 (2009).

36. S. Choubey, W. Rodejohann, and P. Roy, Nucl. Phys. B 808, 272 (2009).

37. S. Dev, S. Kumar, and S. Verma, Phys. Rev. D 79, 033011 (2009).

38. G. Ahuja, M. Gupta, M. Randhawa, and R. Verma, Phys. Rev. D 79, 093006 (2009).

39. S. Goswami, S. Khan, and W. Rodejohann, Phys. Lett. B 680, 255 (2009).

40. E.I. Lashin and N. Chamoun, Phys. Rev. D 80, 093004 (2009).

41. S. Dev, S. Verma, and S. Gupta, Phys. Lett. B 687, 53 (2010).

42. S. Dev, S. Gupta, and R.R. Gautam, Phys. Rev. D 82, 073015 (2010).

43. W. Grimus and P.O. Ludl, Phys. Lett. B 700, 356 (2011).

44. H. Fritzsch, Z.Z. Xing, and S. Zhou, JHEP 1109, 083 (2011).

45. P.O. Ludl, S. Morisi, and E. Peinado, Nucl. Phys. B 857, 411 (2012).

46. D. Meloni and G. Blankenburg, arXiv:1204.2706

47. Z.Z. Xing, Phys. Rev. D 68, 053002 (2003).

48. Z.Z. Xing, Phys. Rev. D 69, 013006 (2004). 
49. A. Merle and W. Rodejohann, Phys. Rev. D 73, 073012 (2006).

50. Y. BenTov and A. Zee, Phys. Rev. D 84, 073012 (2011).

51. E.I. Lashin and N. Chamoun, Phys. Rev. D 85, 113011 (2012).

52. C. Hagedorn and W. Rodejohann, JHEP 0507, 034 (2005).

53. K.R. Deines, E. Dudas, and T. Gherghetta, Nucl. Phys. B 557, 25 (1999).

54. N. Arkani-Hamed, S. Dimopoulos, G. Dvali, and J. March-Russell, Phys. Rev. D 65, 024032 (2001).

55. G.C. Branco and G. Senjanovic, Phys. Rev. D 18, 1621 (1978).

56. D. Chang and R. N. Mohapatra, Phys. Rev. Lett. 58, 1600 (1987).

57. P.Q. Hung, Phys. Rev. D 59, 113008 (1999).

58. P.Q. Hung, arXiv:hep-ph/0006355

59. P.H. Gu and H.J. He, JCAP 0612, 010 (2006).

60. P.H. Gu and U. Sarkar, Phys. Rev. D 77, 105031 (2008).

61. S. Kanemura, T. Nabeshima, and H. Sugiyama, Phys. Lett. B 703, 66 (2011).

62. P.H. Gu, arXiv:1209.4579.

63. G.L. Fogli, E. Lisi, A. Marrone, D. Montanino, A. Palazzo, and A.M. Rotunno, Phys. Rev. D 86, 013012 (2012).

64. D.V. Forero, M. Tórtola, and J.W.F. Valle, Phys. Rev. D 86, 073012 (2012).

65. M.C. Gonzalez-Garcia, M. Maltoni, J. Salvado, and T. Schwetz, JHEP 1212, 123 (2012).

66. C. Jarlskog, Phys. Rev. Lett. 55, 1039 (1985).

67. D.D. Wu, Phys. Rev. D 33, 860 (1986).

68. J.W. Mei and Z.Z. Xing, Phys. Rev. D 69, 073003 (2004).

69. C. Hagedorn, J. Kersten, and M. Lindner, Phys. Lett. B 597, 63 (2004).

70. P.H. Chankowski and Z. Pluciennik, Phys. Lett. B 319, 312 (1993).

71. K.S. Babu, C.N. Leung, and J.T. Pantaleone, Phys. Lett. B 319, 191 (1993).

72. M. Lindner, M. Ratz, and M.A. Schmidt, JHEP 0509, 081 (2005).

73. Z.Z. Xing, Phys. Lett. B 633, 550 (2006).

74. Z.Z. Xing and H. Zhang, Commun. Theor. Phys. 48, 525 (2007).

75. S. Luo, Phys. Rev. D 85, 013006 (2012).

76. S. Luo and Z.Z. Xing, Phys. Rev. D 86, 073003 (2012).

77. Z.Z. Xing and S. Zhou, Neutrinos in Particle Physics, Astronomy and Cosmology (Springer-Verlag, Heidelberg Berlin, 2011).

78. S. Hannestad, A. Mirizzi, G.G. Raffelt, and Y.Y.Y. Wong, JCAP 1008, 001 (2010).

79. W. Rodejohann, Int. J. Mod. Phys. E 20, 1833 (2011). 

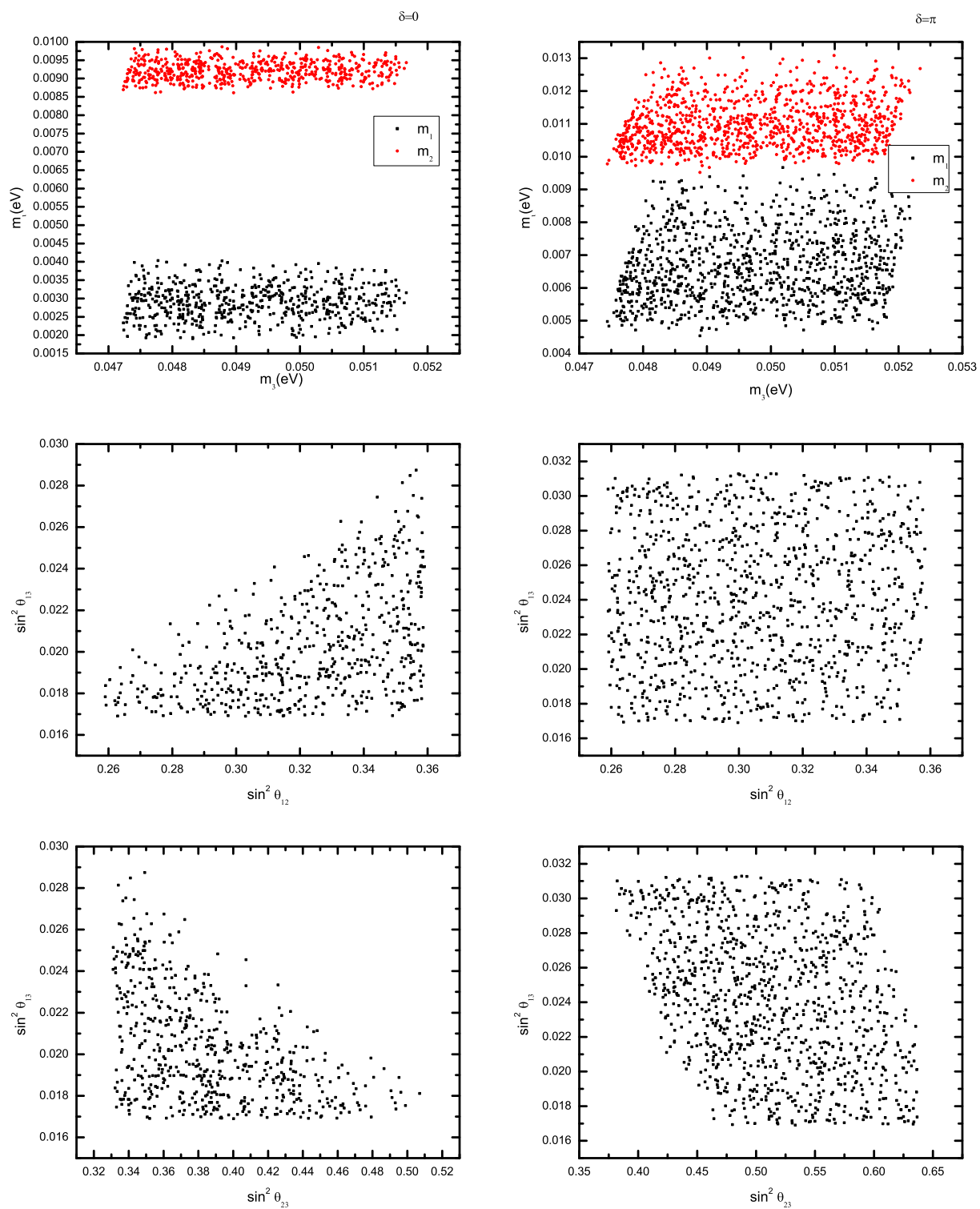

Fig. 1. Pattern $\mathbf{A}_{1}$ of $M_{\nu}$ : The allowed regions of neutrino masses $\left(m_{1}, m_{2}, m_{3}\right)$ and neutrino mixing angles $\left(\sin ^{2} \theta_{12}, \sin ^{2} \theta_{23}, \sin ^{2} \theta_{13}\right)$, where the left column is for $\delta=0$ while the right column for $\delta=\pi$. The global-fit data of two neutrino mass-squared differences $\left(\delta m^{2}, \Delta m^{2}\right)$ and three neutrino mixing angles $\left(\theta_{12}, \theta_{23}, \theta_{13}\right)$ at the $3 \sigma$ level have been input. 

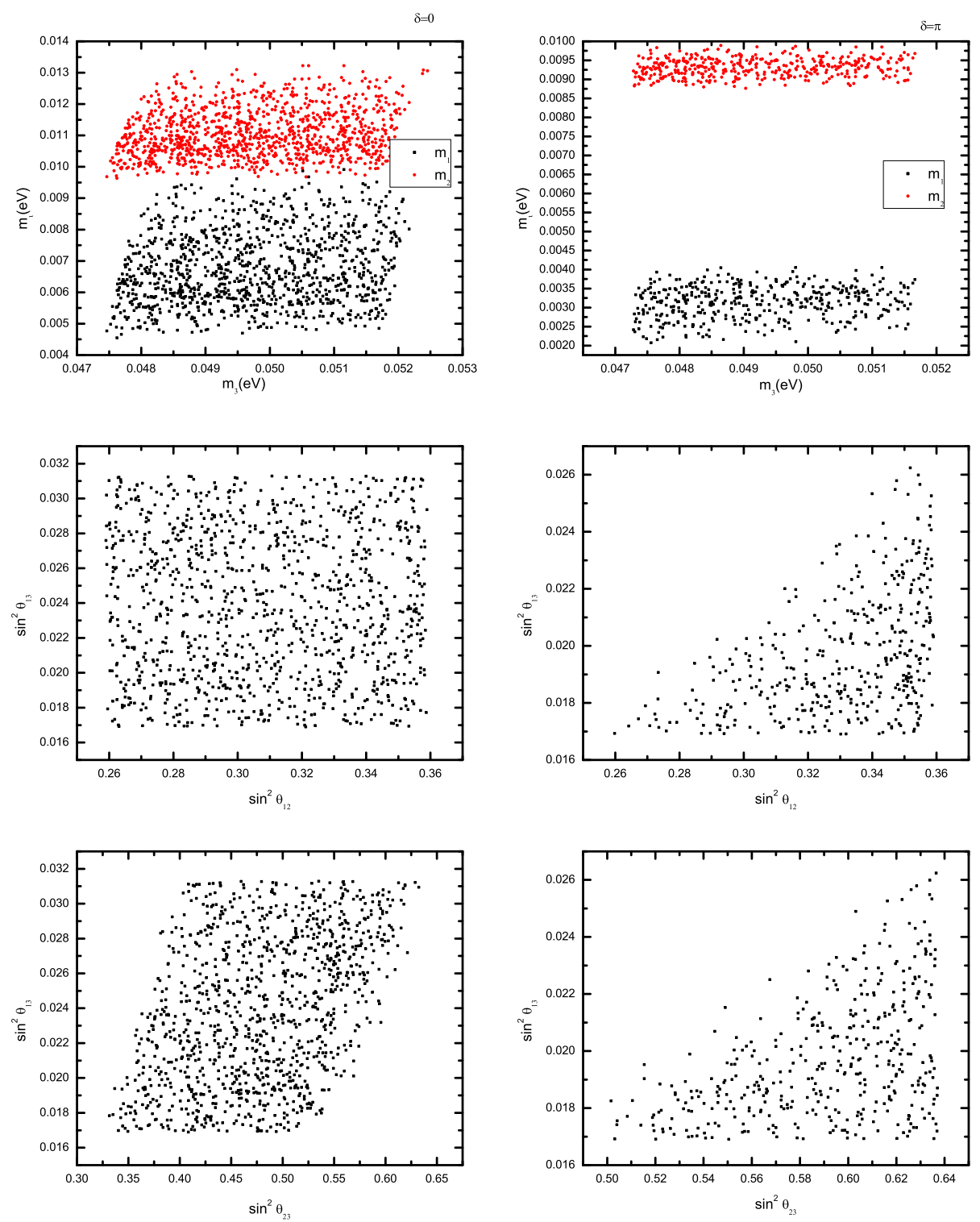

Fig. 2. Pattern $\mathbf{A}_{2}$ of $M_{\nu}$ : The allowed regions of neutrino masses $\left(m_{1}, m_{2}, m_{3}\right)$ and neutrino mixing angles $\left(\sin ^{2} \theta_{12}, \sin ^{2} \theta_{23}, \sin ^{2} \theta_{13}\right)$, where the left column is for $\delta=0$ while the right column for $\delta=\pi$. The global-fit data of two neutrino mass-squared differences $\left(\delta m^{2}, \Delta m^{2}\right)$ and three neutrino mixing angles $\left(\theta_{12}, \theta_{23}, \theta_{13}\right)$ at the $3 \sigma$ level have been input. 

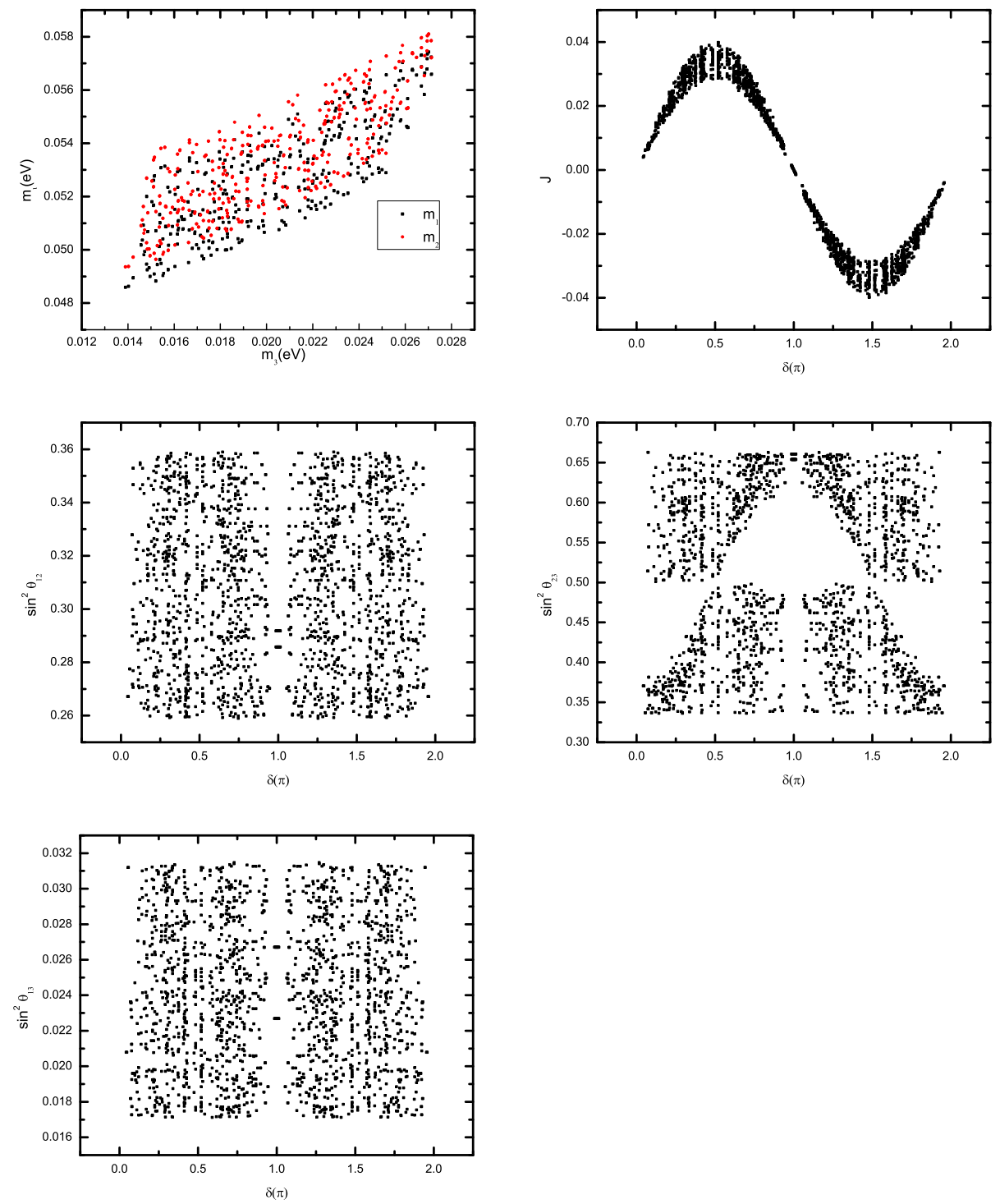

Fig. 3. Pattern $\mathbf{C}$ of $M_{\nu}$ : The allowed regions of neutrino masses $\left(m_{1}, m_{2}, m_{3}\right)$, neutrino mixing angles $\left(\sin ^{2} \theta_{12}, \sin ^{2} \theta_{23}, \sin ^{2} \theta_{13}\right)$, and the Jarlskog invariant versus the CP-violating phase $(J, \delta)$. The global-fit data of two neutrino mass-squared differences $\left(\delta m^{2}, \Delta m^{2}\right)$ and three neutrino mixing angles $\left(\theta_{12}, \theta_{23}, \theta_{13}\right)$ at the $3 \sigma$ level have been input, while the CP-violating phase $\delta$ is allowed to freely vary in $[0,2 \pi)$. 